\title{
Intorno ad una classe notevole di cicli formati da quattro trasformazioni di Laplace nello spazio ordinario.
}

\author{
Memoria di H. JunAs (a Berlino). \\ Alla memoria di Lujei Bianchi \\ nel decimo anniversario della sua morte.
}

\section{Preliminari.}

Preliminari. - Un ciclo di LAPLAck, nell' accennato senso ristretto che varrà pel seguito, comprende quattro superficie su cui si corrispondono i doppii sistemi $(\alpha, \beta)$ di curve fra loro coniugate, le rispettive tangenti, non omologhe s'intende, coincidendo a due a due secondo l'ordine del ciclo e formando così un quadrilatero sghembo variabile. Saranno dette, per brevità, reti opposte quelle descritte dai vertici opposti, le rette che li congiungono chiamandosi diagonali del ciclo. Nel presente lavoro riprendo a trattare la classe particolarmente interessante, già segnalata come un primo esempio nel $1922\left(^{1}\right)$, dei cicli di LAPLACE connessi alla deformazione del paraboloide iperbolico equilatero, e che viene caratterizzata dal distendersi le reti opposte dell' una coppia sopra una medesima sfera, donde intanto, con nna proiettività, si può passare ad una quadrica generale a punti ellittici (i). Tale ciclo di LAPLACE si genera in modo intuitivo nel rotolamento del paraboloide $z=x y$ su una sua deformata, tirandosi dal punto fisso $O$ i raggi paralleli tanto all'asse trascinato nel movimento come al suo simmetrico rapporto al piano tangente. Questi sulla sfera rappresentativa di centro $O$, segnano due sistemi ortogonali $(\alpha, \beta)$, corrispondenti alle asintotiche della deformata ed aventi a comune i loro trasformati di LAPLAOF, talchè viene a nascerne il ciclo desiderato. Giova notar qui che il sistema differenziale da me preso a base analitica dell' indagine è dovuto al DarBoux $\left({ }^{3}\right)$, il quale lo diede risolvendo un altro problema, bensì congenere, senza constatarne l'intimo legame colla

(1) JoNAs, Untersuchungen übeq die als Gewebe beaeidneten Kurvennetze und über eime Reihe von Problemen, die mit de; Verbiegung des gleichseitigen hyperbolischeu Paraboloids zusammenhängen, "Math. Annalen», 87 (1922), p. 157.

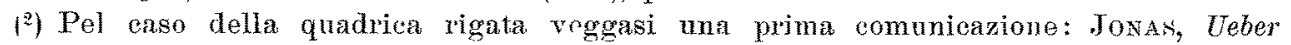
den Zusammenhang zveier Klassen viergliedriger Laplacescher Zyk7en. "Journal f. d. reine u. ang. Math. ", 179 (1938), p. 22.

(3) Darboux, Legons sur la theorie gén. des surf., 3, p. t71. 
deformazione paraboloide. Io vi sono poi ritornato più voltee, fra l'altro per dedurne sotto un nuovo aspetto la teoria delle trasformazioni $B_{k}$ di Braxom per le superficie applicabili sul detto paraboloide $\left({ }^{4}\right)$. L'argomento d'un ulteriore studio recente, riguardante il comportarsi dell' associato ciclo di LAPLACE nell' applicazione della $B_{k}$, mi fu porto dalla scoperta di un teorema generale che il sig. Backes è stato il primo ad enunciare (j), la di lui pubblicazione avendo preceduto di poco la mia propria indipendente ( $\left.{ }^{6}\right)$. Un ciclo di LaPLACE cioè gode della proprietà che le sue diagonali costituiscono due congruenze $W$, nelle quali le asintotiche delle falde focali (reali od immaginarie coniugate) corrispondono alle reti $(\alpha, \beta)$ del ciclo. Partendo da questo fatto fondamentale, ho mostrato che un ciclo della classe speciale in considerazione, se è assoggettato all operazione $B_{k}$, si trasforma in maniera tale che le congruenze $W$ formate dalle diagonali subiscono trasformazioni asintotiche simultanee delle falde focali. Sorse di qui spontanea la domanda se tali trasformazioni potevano estendersi ai cicli di LAPLACE in generale; il che giunsi a stabilire in una seguente Memoria (7), adattando a questo scopo le trasformazioni armoniche delle reti e la nozione di rete derivata.

In quanto all' attuale ricerca, avvertasi prima che nel ciclo di LAPLACE legato alla deformata del paraboloide $z=x y$ vi è corrispondenza fra le sviluppabili delle due congruenze di diagonali, queste ultime essendo polari reciproche rispetto alla sfera cui può sostituirsi, come già si disse, una qua. drica. Faremo vedere che la pensata proprietà riesce caratteristica pei cicli di LAPLACE aventi due reti opposte su una stessa quadrica. Dopo di ciò, tornando al caso della sfera per studiare nuovamente l' effetto del processo $B_{k}$, discorreremo della stratificabilitò in un senso ossia milaterale, avente luogo tra le congruenze $\Gamma$ e $C$ delle diagonali, il raggio generico di $\Gamma$, intersezione dei due piani tangenti alla sfera, ammettendo una semplice infinità di punti, per le superficie luogo dei quali i piani tangenti formano fascio col raggio di $C$, corda di contatto, com'asse. Rammentiamo che secondo Fubrni $\left(^{8}\right)$,

(4) JoNas, Ricerche sulle trasformazioni delle superficie applicabili sul paraboloide iperbolico equilatero, "Annali di Mat. ». (4) 2 (1924-25), p. 161.

(1) BAOKEs, Sur les réseaux conjugués qui se reproduisent après quatre transformations de Laplace, «Bull. Ac. Roy. de Belg. », (5) 21 (1935), p. 883.

(6) Jonas, Ein allgemeiner Satz über W-Kongruenzen mit Anvendungen auf Lapla. cesche Zyklen, Biegungsfächen des einschaligen Hyperboloids und schiefe Weingartensche Systeme, « Math. Annalen », 114 (1937), p. 237.

(i) Jonas, Allgemeine Transfornationstheorie der konjugierten Systeme mit viergliedrigen Taplaceschen Zyklen, "Math. Annalen», 114 (1987). p. 749.

(1) EnmI, Su alcune classi di congruenze di rette e sulle trasformazioni delle super' ficie $R$, "Amali di Mat. ", (4) 1 (1923-24), p. 241. 
mantenuta l'ipotesi che si corrispondano le sviluppabili, una siffatta relazione geometrica vige quando inoltre i piani focali della prima congruenza contengono i fuochi non omologhi della seconda. Orbene nel caso nostro presentasi il fatto assai singolare che tra le $\infty$ 'snperficie della stratificazione ne esistono due segnalate, aventi le $(\alpha, \beta)$ per linee asintotiche, e che si trasformano appunto come le falde focali della congruenza $\Gamma$ per trasformazioni asintotiche. É da osservarsi che la proprietà in questione sta fuor di quanto consegue dalla stratificabilità bilaterale da cui si trovano vincolate la $\Gamma$ e la sua tra. sformata $\Gamma_{1}$, essendo congruenze $R$ nelle condizioni indicate dal FuBrnI $\left({ }^{9}\right)$, cioè contigue per trasformazioni asintotiche delle falde focali. Dopo trattenutici su questa stratificazione per esaminarne più da vicino l'assetto analitico, procediamo a determinare le più generali superficie che abbiano i loro punti situati sui raggi della congruenza $\Gamma$ e prendano parte nel modo di sopra alla trasformazione. Sebbene, propriamente, tale problema pure rientri nella teoria generale delle congruenze $R$, le particolari circostanze che ne accompagnano la risoluzione appaiono degne d'attenzione, tanto più che c'imbattiamo così in un sistema difierenziale, generalizzazione di quello di DARBoux, al quale può ridursi, per conveniente scelta dei valori iniziali, la deformazione del paraboloide rigato generale. Anzi, con ciò, saremo in grado di dire da qual ente geometrico venga surrogato allora il ciclo di LAPLACE. Rimandando pei dettagli alle righe che seguono, basti qui avvertire che i nuovi sistemi di quadrilateri sghembi risultano identici a quelli ottenuti risolvendo il problema con cui termineremo la nostra ricerca, di trovare cioè le coppie di doppii sistemi di linee $(\alpha, \beta)$, appartenenti ad una medesima sfera, $\theta$ per cui si confondano i piani osculatori alle curve non omologhe nei punti corrispondenti.

1. Generalità sui cicli di Laplace. - Comincio coll' esporre in succinto il metodo metrico di eui mi sono servito per la ricerca dei cicli di LAPLACE in due Memorie anteriori $\left({ }^{10}\right)$. Indichino $x$ (cioè $\left.x, y, z\right)$ e $x^{t}$ i punti descriventi due opposte delle quattro reti $(\alpha, \beta)$ del ciclo domandato, essendo

$$
x_{\alpha \beta}=a x_{x}+b x_{\beta}, \quad x_{\alpha \beta}^{\prime}=a^{\prime} x_{\alpha}^{\prime}+b^{\prime} x_{\beta}^{\prime}
$$

le relative equazioni di LAPLAcE. Sarà necessario in primo luogo ehe, posto;

$$
a-\frac{b_{\beta}}{b}=A, \quad b-\frac{a_{\alpha}}{a}=B, \quad a^{\prime}-\frac{b_{\beta}^{\prime}}{b^{\prime}}=A^{\prime}, \quad b^{\prime}-\frac{a_{\alpha}^{\prime}}{a^{\prime}}=B^{\prime},
$$

(9) Ibid.

(10) Cf. il $\& 3$ della Mem. citata in nota $\left(^{6}\right)$. 
le quattro funzioni $a, b, a^{\prime}, b^{\prime}$ soddisfino alle condizioni seguenti :

$$
A_{\alpha}+b A=B_{\beta}^{\prime}+a^{\prime} B^{\prime}=A B^{\prime}, \quad B_{\beta}+a B=A_{\alpha}^{\prime}+b^{\prime} A^{\prime}=B A^{\prime} .
$$

In virtù di queste risultano illimitatamente integrabili le seguenti equazioni a derivate parziali (delle quali la seconda della (1) è conseguenza):

$$
\left\{\begin{array}{l}
x_{x}^{\prime}=\frac{b^{\prime}}{a} x_{\beta}+b^{\prime}\left(x^{\prime}-x\right), \quad x_{\beta}^{\prime}=\frac{a^{\prime}}{b} x_{\alpha}+a^{\prime}\left(x^{\prime}-x\right), \\
x_{\alpha \alpha}=\left(b+\frac{b_{\alpha}}{b}+B^{\prime}\right) x_{\alpha}+b B^{\prime}\left(x^{\prime}-x\right), \\
x_{\alpha \beta}=a x_{\alpha}+b x_{\beta}, \\
x_{\beta \beta}=\left(a+\frac{a_{\beta}}{a}+A^{\prime}\right) x_{\beta}+a A^{\prime}\left(x^{\prime}-x\right),
\end{array}\right.
$$

le quali, riguardate come sistema lineare ed omogeneo per la terna di funzioni incognite $x_{\alpha}, x_{\beta}, x^{\prime}-x$, posseggono tre soluzioni linearmente indipen. denti, in guisa da definire un ciclo di LAPLACE in modo univoco a meno di trasformazioni affini. Per le reti intermedie $(\widehat{x})$ e $(\bar{x})$, trasformate comuni di LAPLACE delle superficie $(x)$ e $\left(x^{\prime}\right)$, avremo:

$$
\begin{gathered}
\bar{x}=x-\frac{x_{\alpha}}{b}=x^{\prime}-\frac{x_{\beta}^{\prime}}{a^{\prime}}, \quad \bar{x}=x-\frac{x_{\beta}}{a}=x^{\prime \prime}-\frac{x_{x}^{\prime}}{b^{\prime}}, \\
\widehat{x}_{\alpha \beta}=A \bar{x}_{\alpha}+B^{\prime} \bar{x}_{\beta}, \quad \bar{x}_{x_{\beta} \beta}=A^{\prime} \bar{x}_{z}+B \bar{x}_{\alpha} .
\end{gathered}
$$

Dalle (2), (3) deduconsi le formule ausiliarie, utili più tardi :

$$
\begin{gathered}
\left(a+a^{\prime}+A+A^{\prime}\right)_{\alpha}=\left(b+b^{\prime}+B+B^{\prime}\right)_{a} \\
\left(a a^{\prime} A A^{\prime}\right)_{x}=0, \quad\left(b b^{\prime} B B^{\prime}\right)_{\beta}=0 .
\end{gathered}
$$

A proposito della relazione (7) notisi che, introducendo il determinante:

$$
\Delta=\left|\begin{array}{lll}
x_{\alpha} & x_{\beta} & x^{\prime}-x \\
y_{x} & y_{\beta} & y^{\prime}-y \\
z_{\alpha} & z_{\beta} & z^{\prime}-z
\end{array}\right|
$$

viene:

$$
\left(\log \frac{\Delta}{a b}\right)_{x}=b+b^{\prime}+B+B^{\prime}, \quad\left(\log \frac{\Delta}{a b}\right)_{\beta}=a+a^{\prime}+A+A^{\prime} .
$$

D'altra parte, in virtù delle (8), come facilmente confermasi col tener conto della forma del sistema differenziale (4), sari sempre possibile disporre delle variabili $\alpha$ e $\beta$ in modo da aversi con $\varepsilon_{1}, \varepsilon_{2}$ unita positive o negative:

$$
a a^{\prime} A A^{\prime}=\varepsilon_{1}, \quad b b^{\prime} B B^{\prime}=\varepsilon_{2} \text {. }
$$


Ricordiamo ancora l'importante proprietà dei cicli di LAPLACE che le diagonali $x x^{\prime}$ e $\bar{x} \bar{x}$ formano due congruenze $W$ con $\alpha, \beta$ parametri asintotici delle falde focali, le sviluppabili determinandosi rispettivamente dalle equazioni differenziali :

$$
\frac{d \beta}{d \alpha}= \pm \sqrt{\frac{\overline{b b^{\prime}}}{a a^{\prime}}}, \quad \frac{d \beta}{d \alpha}= \pm \sqrt{\frac{\overline{B B^{\prime}}}{A A^{\prime}}}
$$

Per una ben nota proposizione di DABBoux in ogni eongruenza $W$ le sei coordinate di retta soddisfano ad un' equazione di LAPLACE. Questa per la congruenza delle rette $x x^{\prime}, \operatorname{con} \theta=x^{\prime}-x, \ldots, y z^{\prime}-z y^{\prime}, \ldots$, assume la forma:

$$
\theta_{\alpha \beta}=\left(a+a^{\prime}\right) \theta_{\alpha}+\left(b+b^{\prime}\right) \theta_{\beta}-\left(a b^{\prime}+a^{\prime} b\right) \theta_{\text {. }}
$$

valendo l'analoga per quella delle rette $\widehat{x} \vec{x}$ :

$$
\theta_{x \beta}=\left(A+A^{\prime}\right) \theta_{x}+\left(B+B^{\prime}\right) \theta_{\beta}-\left(A B+A^{\prime} B^{\prime}\right) \theta .
$$

2. Una proprietà caratteristica dei cicli di Laplace con due reti opposte sopra una medesima quadrica. - Ci proponiamo ora di dimostrare il teorema seguente: Se in un ciclo di LAPLACE ha luogo corrispondenza fra le sviluppabili delle due congruenze $\mathrm{W}$ formate dalle diagonali, le veti opposte dell una coppia appartengono ad una medesima quadrica.

Poichè, nell' attuale ipotesi, dalla (12) si ha:

$$
\frac{b b^{\prime}}{a a^{\prime}}=\frac{B B^{\prime}}{A A^{\prime}}
$$

ammesse le (11) e cioè per causa di realità con $\varepsilon_{1}=\varepsilon_{2}$, si ottiene:

$$
\frac{b b^{\prime}}{a \alpha^{\prime}}=\frac{B B^{\prime}}{A A^{\prime}}= \pm 1
$$

Sarà lecito, per semplificare la scrittura e senza pregiudicare l'esattezza dell' argomentazione, supporre reali nelle due congruenze $W$ le sviluppabili, date allora da:

$$
\not z \pm \beta=\text { cost. }
$$

Avendosi dunque

$$
a a^{\prime}=b b^{\prime}, \quad A A^{\prime}=B B^{\prime}, \quad a a^{\prime} A A^{\prime}=b b^{\prime} B B^{\prime}=\varepsilon= \pm 1,
$$

poniamo per un momento:

$$
a a^{\prime}=b b^{\prime}=p .
$$

Derivando coll' aver riguardo alla (2) troviamo:

$$
\frac{\rho_{x}}{\rho}=b+b^{\prime}-\left(B+B^{\prime}\right), \quad \frac{\rho_{\beta}}{\rho}=a+a^{\prime}-\left(A+A^{\prime}\right) .
$$


indi dal confronto colla (7):

$$
\left(a+\alpha^{\prime}\right)_{x}=\left(b+b^{\prime}\right)_{\beta}, \quad\left(A+A^{\prime}\right)_{x}=\left(B+B^{\prime}\right)_{\beta} .
$$

Ł manifesto che nelle due congruenze $W$ le curve $\alpha \pm \beta=$ cost, intercettate dalle sviluppabili su ciascuna delle falde focali (anch'esse reali com'è ovvio). costituiscono un sistema isotermo-coniugato; ne segue che le diagonali $\mathbf{x x}^{\prime}$ e $\overline{\mathbf{x}} \overline{\mathrm{x}}$ percorrono congruenze $\mathrm{R}$. A conferma di cio si osservi altresi che per le (17) le equazioni di LAPLACE (13), (14) risultano ad invarianti uguali. Segnaliamo di passaggio una proposizione generale che si deduce dalla sola (7): Se in un ciclo di LAplace una delle due congruenze di diagonali è $\mathrm{R}$, la proprietò sussiste per l'altra. Tuttavia tale ipotesi sarebbe diversa dalla nostra, non discendendone generalmente la corrispondenza delle sviluppabili.

Eseguite le derivazioni nelle (17) coll' uso di (2), (3), otteniamo la seguente equazione in termini finiti da agginngersi alla (16):

$$
a B-b A+a^{\prime} B^{\prime}-b^{\prime} A^{\prime}=0 .
$$

la quale, eliminando p. es. $a^{\prime}$ e $A^{\prime}$, viene a scriversi :

$$
(a B-b A)\left(a A-b^{\prime} B^{\prime}\right)=0 .
$$

Resta con ciò spiegato il differente comportamento delle due coppie di reti opposte nel ciclo ricercato. Abbiamo in conseguenza le relazioni:

$$
\frac{a}{b}=\frac{b^{\prime}}{a^{\prime}}=\frac{A}{B}=\frac{B^{\prime}}{A^{\prime}}
$$

ovvero:

$$
\frac{a}{\bar{b}^{\prime}}=\frac{b}{a^{\prime}}=\frac{A^{\prime}}{\bar{B}}=\frac{B^{\prime}}{A}
$$

completata dalla terza delle (16). Basta ora, come si vede senz'altro, che prendiamo a fondamento degli ulteriori sviluppi l'uno di questi gruppi di formule, p. es. le (19), l'altro corrispondendo allora soltanto ad uno seambio delle due coppie di reti opposte.

Combinando le (19) colle (2) avremo:

$$
a_{\alpha}=b_{\beta}, \quad a_{\alpha}^{\prime}=b_{\beta}^{\prime},
$$

onde appare che le reti $(\alpha, \beta)$ descritte dai punti $x$ ə $x^{\prime}$ saranno ad invarianti

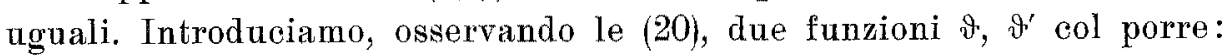

$$
a=-\frac{\mathfrak{F}_{\beta}}{\mathscr{V}^{\prime}}, \quad b=-\frac{\mathfrak{\vartheta}_{\alpha}}{\mathfrak{W}}, \quad a^{\prime}=-\frac{\mathscr{V}_{\beta}^{\prime}}{\mathscr{W}^{\prime}}, \quad b^{\prime}=-\frac{\mathscr{\vartheta}_{\alpha}^{\prime}}{\mathscr{W}^{\prime}} .
$$


Deve aversi $a a^{\prime}=b b^{\prime}$, ciò̀ $\vartheta_{\alpha} q^{\prime}{ }_{\alpha}=\vartheta_{\beta}^{\dagger} \vartheta_{\beta}{ }^{\prime}$, onde potremo fare:

$$
w_{x}^{\prime}=\frac{1}{h} \mathfrak{w}_{\beta}, \quad w_{\beta}=\frac{1}{h} \hat{y}_{\alpha} \text {. }
$$

Dalle (21) si ricavano per mezzo delle (2) le espressioni:

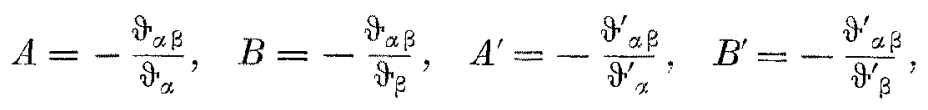

compatibili peraltro colle rimanenti (19). Ci resta adesso da esprimere che $\mathrm{i}$ valori di $a, b, a^{\prime}, b^{\prime}$ dedotti dalle (23) mediante le (3) devono identificarsi colle formule (21). Per questo, presa la relazione.

$$
\frac{A_{x}}{A}+b-B^{\prime}=0
$$

e sostituitevi per $b$ e $B^{\prime}$ le espressioni (21) e (23), scriviamo:

$$
\frac{A_{\alpha}}{A}-\frac{\mathfrak{W}_{\alpha}}{\mathfrak{Y}}+\frac{\mathscr{W}_{\alpha \ell}^{\prime}}{\mathfrak{W}_{\beta}^{\prime}}=0
$$

indi integrando ed unendovi le formule analoghe:

$$
A \frac{\mathscr{V}_{\beta}^{\prime}}{\vartheta^{\prime}}=f_{1}(\beta), \quad B \frac{\vartheta^{\prime} \alpha}{\vartheta^{\prime}}=f_{2}(\alpha), \quad A^{\prime} \frac{\vartheta_{\beta}}{g^{\prime}}=f_{3}(\beta), \quad B^{\prime} \frac{\vartheta_{\alpha}}{\vartheta^{\prime}}=f_{4}(\alpha)
$$

Tenendo presenti le (23), si avrà:

$$
\frac{q_{\alpha \beta}}{h q^{2}}=-f_{1}(\beta)=-f_{2}(\alpha)=\mathrm{cost}, \quad \frac{h \vartheta^{\prime} \beta}{q^{\prime}}=-f_{3}(\beta)=-f_{4}(\alpha)=\mathrm{cost} \text {. }
$$

Delle due costanti così introdotte l' una e cioè p. es. la prima potrì ridursi all' unita positiva, munendo a tal fine le funzioni of e $h$ dello stesso moltiplicatore costante opportunamente scelto, in guisa da lasciar inalterate le (22), mentre allora la seconda costante per effetto della terza formula (16), con cio verificata, riesce identica alla $\varepsilon= \pm 1$ che vi figura. Essendo:

$$
\begin{gathered}
\mathscr{\vartheta}_{\alpha \beta}=h \vartheta, \quad \mathscr{\vartheta}_{\alpha \beta}^{\prime}=\frac{\varepsilon}{h} \vartheta^{\prime}, \\
A=-\frac{h \vartheta}{\vartheta_{x}}, \quad B=-\frac{h \vartheta}{\vartheta_{\beta}}, \quad A^{\prime}=-\varepsilon \frac{\vartheta^{\prime}}{h \vartheta^{\prime}{ }_{x}}, \quad B^{\prime}=-\varepsilon \frac{\vartheta^{\prime}}{h \vartheta_{\beta}^{\prime}},
\end{gathered}
$$

bisogna infine paragonare colla seconda (24) i due valori di $\mathfrak{F}_{\alpha \beta}^{\prime}$ dedotti dalle (22). Siamo cosi pervenuti al sistema differenziale:

$$
\left\{\begin{array}{l}
\vartheta_{\alpha}^{\prime}=\frac{1}{h} \vartheta_{\beta}, \quad \vartheta_{\beta}^{\prime}=\frac{1}{h} \vartheta_{\alpha}, \\
\vartheta_{x \alpha}=\frac{h}{h} \vartheta_{\alpha}+\varepsilon \vartheta^{\prime}, \quad \vartheta_{\alpha \beta}=h \vartheta_{;} ; \quad \vartheta_{\beta \beta}=\frac{h}{h} \vartheta_{\beta}+\varepsilon \vartheta^{\prime}, \quad(\varepsilon= \pm 1)
\end{array}\right.
$$


ove alle equazioni della seconda riga si potrebbero sostituire le equivalenti:

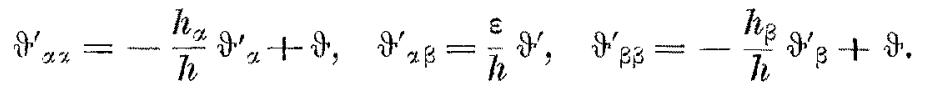

Alle (20) riconosciamo associarsi in ogni caso la condizione:

$$
(\log h)_{\% ; \beta}=h-\frac{\varepsilon}{h},
$$

soddisfatta la quale il sistema differenziale risulta illimitatamente integrabile. In quanto alla (28), trascurando qui le particolarita di geometria immaginaria, ci contentiamo di ricordare che essa in sostanza non è attro che l'equazione differenziale delle superficie a curvatura costante.

Assumendo ora le terne $\xi, \eta, \zeta$ e $\xi^{\prime}, \eta^{\prime}$, $\zeta^{\prime}$ col porre:

$$
x=\frac{\xi}{9}, \quad y=\frac{\eta}{y}, \quad z=\frac{\zeta}{9}, \quad x^{\prime}=\frac{\xi^{\prime}}{y^{\prime}}, \quad y^{\prime}=\frac{\eta^{\prime}}{\vartheta^{\prime}}, \quad z=\frac{\zeta^{\prime}}{\vartheta^{\prime}},
$$

avremo invece delle formule (5):

$$
\ddot{x}=\frac{\xi_{x}}{\hat{\vartheta}_{x}}=\frac{\vec{\xi}_{\beta}^{\prime}}{\hat{V}_{\beta}^{\prime}}, \quad \widetilde{x}=\frac{\xi_{\beta}}{\hat{\vartheta}_{\beta}}=\frac{\xi_{x}^{\prime}}{\hat{\vartheta}_{x}^{\prime}},
$$

mentre il sistema differenziale (4) verrà a mutarsi appunto nel sistema (26) soddisfatto da $\vartheta$, $\vartheta^{\prime}$, valendo così adunque per le quattro coppie di funzioni $\xi, \xi^{\prime} ; \eta, \eta^{\prime} ; \zeta, \zeta^{\prime} ; \vartheta, \vartheta^{\prime}$. Considerandolo come lineare e completo nelle funzioni incognite $\vartheta, \vartheta^{\prime}, \vartheta_{x}, \vartheta_{\beta}$, riesce facile a vedersi che esso ammette quattro soluzioni linearmente indipendenti, onde il determinante

$$
\delta=\left|\begin{array}{llll}
\xi & \xi^{\prime} & \xi_{x} & \xi_{p} \\
\eta & \eta^{\prime} & \eta_{x} & \eta_{\beta} \\
\zeta & \zeta^{\prime} & \zeta_{x} & \zeta_{\beta} \\
\vartheta & \vartheta^{\prime} & \vartheta_{\alpha} & \vartheta_{p}
\end{array}\right|
$$

sarà diverso da zero. Si verifica per derivazione il sussistere di un gruppo di dieci relazioni integrali quadratiche, presentanti i due tipi:

$$
\left\{\begin{array}{l}
\frac{2}{h} \xi_{\alpha} \xi_{\beta}-\xi^{2}-\varepsilon \xi^{\prime 2}=c_{i 1}, \\
\frac{1}{h}\left(\xi_{\alpha} \eta_{\beta}+\xi_{\beta} \eta_{\alpha}\right)-\xi_{\eta}-\varepsilon \xi^{\prime} \eta^{\prime}=c_{12} .
\end{array}\right.
$$

Denotiamo con $c_{i k}\left(i, k=1,2,3,4 ; c_{i k}=c_{k i}\right)$ le dieci costanti, intendendosi presi gli indici $1,2,3,4$ nell'ordine delle lettere $\xi, \eta, \zeta, \$$, e formiamone il determinante del $4^{\circ}$ ordine:

$$
\left\|c_{i k}\right\|=c
$$


Quadrando ó, ciò che si fa coll'applicare la consueta regola al determinante indicato dalla prima riga:

$$
\left(\frac{\xi_{\alpha}+\xi_{\beta}}{\sqrt{2 h}}, \frac{i\left(\xi_{\alpha}-\xi_{\xi}\right)}{\sqrt{2 h}}, \quad i \xi, \quad i \sqrt{\overline{2}} \overline{\xi^{\prime}}\right)
$$

e tenendo conto delle (32), otterremo la relazione:

$$
c=-\varepsilon \frac{\delta^{2}}{h^{2}} .
$$

Importa avvertire che, calcolate le $c_{k}$ mediante le (32) dai valori iniziali delle sedici funzioni $\xi, \xi^{\prime}, \xi_{x}, \xi_{\beta}, \ldots$, arbitrarii pur che ne risulti $\delta \neq 0$, si ha per la (34): $\varepsilon=-\operatorname{sgn} c$.

Indicansi con $C_{1 k}$ i complementi algebrici degli elementi $c_{k, k}$ nel determinante (31), onde verrà:

$$
C=\left\|C_{i k}\right\|=c^{3} \text {. }
$$

Orbene, pex provare che nel quadrilatero sghembo variabile $x: \bar{x} \bar{x}$, generante il ciclo di LAPLACE, i due vertici opposti $\bar{x}$ e $\bar{x}$ appartengono ad una mede. sima quadrica, consideriamo la forma quadrica quaternaria:

$$
\begin{aligned}
& \Phi=C_{11} \xi^{2}+2 C_{12} \xi \eta+2 C_{13} \xi \zeta+2 C_{14} \xi \vartheta \\
& +C_{22} \eta^{2}+2 C_{23} \eta \zeta+2 C_{24} \eta \eta^{2} \\
& +C_{33} \zeta^{2}+2 C_{34} \xi^{2} \\
& \text { +1 } \mathrm{C}_{44} 9^{92} \text {, }
\end{aligned}
$$

la quale seritta nelle $\xi^{\prime}, \ldots$ denoteremo con $\Phi^{\prime}$, essendo inoltre

$$
\text { II }=C_{11} \xi \xi^{\prime}+C_{12}\left(\xi \eta^{\prime}+\eta \xi^{\prime}\right)+\ldots+C_{44} \psi^{2} \psi^{\prime}
$$

la rispettiva forma polare. Se deriviamo la (36) rapporto ad $\alpha$ e $\beta$, giungeremo per effetto delle (32) a stabilire quattro relazioni della forma:

$$
\frac{1}{2 h} \Phi_{\beta} \xi_{\alpha}+\frac{1}{2 h} \Phi_{\alpha} \xi_{\beta}=(\Phi+c) \xi+\varepsilon \Pi \xi^{\prime} .
$$

da eui, avendosi $\delta \neq 0$, si ricavano le seguenti:

$$
\Phi+c=0, \quad \Phi_{\alpha}=0, \quad \Phi_{\beta}=0 . \quad \Pi=0 .
$$

In modo simile si ottiene:

$$
\Phi^{\prime}+\varepsilon c=0, \quad \Phi_{a}^{\prime}=0, \quad \Phi_{\beta}^{\prime}=0 .
$$

Dopo di ciò, derivando le $\Phi_{\alpha}=0, \Phi_{\beta}=0$ un'alra volta e sostituendo per $\xi_{x x}, \ldots . \xi_{p \zeta}, \ldots$ le espressioni date dalle (26), avremo coll' osservare la $\Pi=0$ :

$$
\left\{\begin{array}{l}
C_{11}\left(\xi_{x}\right)^{2}+2 C_{12} \xi_{2} \eta_{\alpha}+\ldots+C_{44}\left(\vartheta_{\alpha}\right)^{2}=0, \\
C_{11}\left(\xi_{\beta}\right)^{2}+2 C_{12} \xi_{\beta} \eta_{\beta}+\ldots+C_{44}\left(\vartheta_{k}\right)^{2}=0 .
\end{array}\right.
$$


Notiamo d'altronde ohe si ricadrà nella prima formula (40), quando si calcoli $\Phi_{\alpha \beta}$ col far uso delle (26) e (33). Ora, il teorema enunciato al principio del presente numero risulta pienamente confermato dalle (41), siccome queste in in virtù delle $(30)$ si convertono nell' equazione della quadrica:

$$
\begin{gathered}
C_{14} \widehat{x}^{2}+2 C_{12} \widehat{x} \hat{y}+2 C_{13} \widehat{x z}+2 C_{14} \hat{x}+C_{2 z} \hat{y}^{2}+2 C_{13} \widehat{y z} \\
+2 C_{24} \bar{y}+C_{33} \bar{z}+2 C_{34} \bar{z}+C_{44}=0 .
\end{gathered}
$$

soddisfatta altresi da $\bar{x}, \bar{y}, \bar{z}$.

Come ben si sà, il segno della curvatura totale della quadrica è quello di $-C$, cioè tenuto conto della relazione (35), di $-c$; onde, osservando la (34), possiamo dire: Secondochè nelle equazioni differenziali $(26),(28)$ è $\varepsilon=+10$ $\varepsilon=-1$, restando pur nell' ipotesi di sviluppabili reali, la quadrica ricoperta dalle due reti opposte $(\overline{\mathrm{x}})$ e $(\overline{\mathrm{x}})$ del ciclo sarà rispettivamente a punti ellittici o rigata. Quanto all' ipotesi fin qui esclusa che nelle congrnenze $W$ delle diagonali le sviluppabili sieno immaginarie coniugate, ci limiteremo ad accennare che allora nell' equazione differenziale (28) della funzione $h$ il segno $\varepsilon$ si collega a quello della enrvatura totale in modo opposto, vale a dire che è $\varepsilon=+1$ nel caso della quadrica rigata.

3. Caso della sfera; deformate del paraboloide iperbolico equilatero; stratificabilità unilaterale delle due congruenze di diagonali. - Passiamo ora a completare i risultati conseguiti nelle mie Memorie precedenti rispetto ai cicli di LAPLAOn per cui due opposte delle quattro superficie coincidono nella stessa sfera unitaria col centro nell' origine. A tale nopo, per maggior chiarezza, scriviamo di nuovo, con $\varepsilon=-\uparrow 1$, le equazioni differenziali :

$$
\begin{gathered}
(\log h)_{\alpha \beta}=h-\frac{1}{h}, \\
\xi_{\alpha}^{\prime}=\frac{1}{h} \xi_{\beta}, \quad \xi_{\beta}^{\prime}=\frac{1}{h} \xi_{\alpha}, \\
\xi_{\alpha x}=\frac{h}{h} \xi_{\alpha}+\xi^{\prime}, \quad \xi_{\alpha \beta}=h \xi, \quad \xi_{\beta \beta}=\frac{h_{\beta}}{h} \xi_{\beta}+\xi^{\prime} \\
{\left[\xi_{\alpha x}=-\frac{h_{\alpha}}{h} \xi_{\alpha}^{\prime}+\xi, \quad \xi_{\alpha \beta}^{\prime}=\frac{1}{h} \xi^{\prime}, \quad \xi_{\beta}^{\prime}=-\frac{h_{\beta}}{h} \xi_{\beta}^{\prime}+\xi\right] .}
\end{gathered}
$$

Data che sia la $h$, supponiamo di conoscere inoltre una coppia di soluzioni $\vartheta$, $\vartheta^{\prime}$ del sistema (44), per la quale la prima relazione (32) prende la forma:

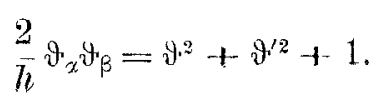


Occorre ricordar qui rapidamente il metodo con cui se ne deduce il sistema completo delle soluzioni. Notiamo che dalla forma differenziale quadratica

$$
\Sigma d \widehat{X}^{2}=\frac{d \alpha^{2}}{\left(\hat{q}_{\alpha}\right)^{2}}+\frac{d \beta^{2}}{\left(\vartheta^{\prime} \beta\right)^{2}}
$$

viene definito l'elemento lineare di una sfera $(\widehat{X})$ di raggio unità riferita alla rete ortogonale $(\alpha, \beta)$; onde se ne deducono per $\xi, \xi$, ... le formule:

$$
\xi=\mathfrak{\vartheta} \widehat{X}+\mathfrak{\vartheta}_{\beta}^{\prime} \widehat{X}_{\beta}, \quad \xi^{\prime}=\mathscr{\vartheta}^{\prime} \widehat{X}+\mathfrak{\vartheta}_{\alpha} \widehat{X}_{\alpha} .
$$

Sono da aggiungersi, col sommatorio esteso alle lettere $\xi, \eta, \zeta$, le relazioni:

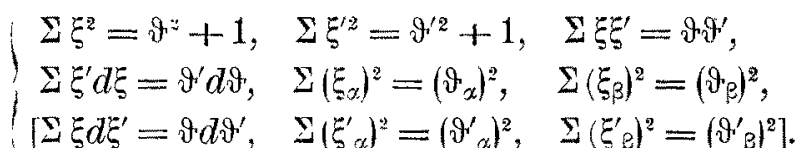

Vale quindi la seguente rappresentazione del ciclo di LAPLACE:

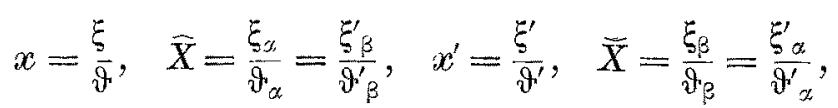

giacendo i punti $\widehat{X}$ e $\bar{X}$ sulla sfera $X^{2}+Y^{2}+Z^{2}=1$.

Al tempo stesso si determina con tre quadrature una superficie

$$
x^{*}=\int\left(\xi^{\prime} d q+\xi d v^{\prime}\right)
$$

applicabile sul paraboloide iperbolico equilatero $z=x y$, avendosi dalle (48):

$$
\Sigma\left(d x^{*}\right)^{2}=d \mathscr{F}^{2}+d \mathscr{V}^{\prime 2}+\left[d\left(\vartheta+\mathscr{V}^{\prime}\right)\right]^{2} .
$$

Sopra di essa le curve $(\alpha, \beta)$ sono le asintotiche. Badisi però che la deformazione, nelle condizioni di realità da noi osservate, riesce di prima specie, cioè a sistema coniugato permanente reale, corrispondendo questo alle sviluppa* bili $\alpha \pm \beta=$ cost delle congruenze di diagonali del ciclo.

Seguendo la convenzione fatta nei preliminari, diremo $\Gamma$ la congruenza $W$ formata dalle rette $x x^{\prime}$ e $C$ quella delle rette $\bar{X} \bar{X}$. Dobbiamo ricordar qui che i fuochi, corrispondentisi fra di loro al modo stabilito dalle sviluppabili, vengono dati dalle formule:

$$
x_{\Gamma}^{(1,2)}=\frac{\xi \pm h \xi^{\prime}}{q \pm h q^{\prime}}, \quad x_{C}^{(1,2)}=\frac{\xi_{\alpha} \pm \xi_{\beta}}{\vartheta_{\alpha} \pm \vartheta_{\beta}} .
$$

Notisi ancora che sulle quattro falde focali le curve $(\alpha, \beta)$ formano le asintotiche, ed anzi che $\mathrm{i}$ piani tangenti alle superficie $\left(x_{\Gamma}^{(1)}\right)$ e $\left(x_{\Gamma}^{(2)}\right)$ contengono 
rispettivamente i punti $x_{C}^{(1)}$ e $x_{C}^{(2 /}$. Ciò premesso, consideriamo sul raggio $x x^{\prime}$ di $\Gamma$ il punto definito da:

$$
x^{[x]}=\frac{\xi+x \xi^{\prime}}{\vartheta+x \vartheta^{\prime}}
$$

con $x$ costante arbitraria. Dico ehe, per ciascuna superficie $(x[x])$ di questa schiera, il piano tangente passa per l'altra diagonale $\bar{X} \widetilde{X}$ del ciclo; il che si prova facilmente coll' uso di coordinate omogenee, essendo:

$$
\left(\left[\xi+x \xi^{\prime}\right]_{\alpha}, \xi+x \xi^{\prime}, \xi_{x}, \xi_{\beta}\right)=0, \quad\left(\left[\xi+x \xi^{\prime}\right]_{\beta}, \xi+x \xi^{\prime}, \xi_{x}, \xi_{\beta}\right)=0 .
$$

Donde: La congruenza $\Gamma$ delle intersezioni $\mathrm{xx}^{\prime}$ dei piani tangenti alla sfera $\dot{e}$ stratificabile in un senso colla $\mathrm{O}$ delle rette $\mathrm{\textrm {X }} \overline{\mathrm{X}}$, corde di contatto.

4. Applicazione della trasformazione $B_{k}$ di Bianchi. - Denotando con $a, b$ costanti vincolate dalla relazione ("):

$$
a^{2}+b^{2}=1
$$

calcoleremo le nuove quaderne $\xi_{1}, \eta_{1}, \zeta_{1}, \vartheta_{1}$ e $\xi_{1}^{\prime}, \ldots$ mediante formule:

$$
\xi_{1}=l \xi_{\alpha}-a \xi-b \xi^{\prime}, \quad \xi_{1}^{\prime}=\frac{2 a b}{t} \xi_{\alpha}^{\prime}-b \xi-a \xi^{\prime}
$$

ove $t$ denoti un integrale del sistema di RICCATI:

$$
t_{\alpha}=-\frac{1}{2 b} t^{2}-\frac{h_{\alpha}}{h} t+a, \quad t_{\beta}=-\frac{h}{2 a} t^{2}+\frac{b}{h} .
$$

Coll' assumere inoltre la funzione

$$
h_{1}=\frac{h t^{z}}{2 a b}
$$

soddisfacente anch' essa l'equazione differenziale (43), risulteranno verificate per l'indice 1 tutte le relazioni del numero precedente. Dal processo analitico $B(a, b)$ così definito, equivalente all'operazione $B_{k}$ di BraNcHI con $k=-2 a b$, avremo il ciclo trasformato:

$$
x_{1}=\frac{\xi_{1}}{\vartheta_{1}}, \quad \bar{X}_{1}=\frac{\left(\xi_{1}\right)_{\alpha}}{\left(\vartheta_{1}\right)_{x}}=\frac{\left(\xi_{1}^{\prime}\right)_{\beta}}{\left(\vartheta_{1}^{\prime}\right)_{\beta}}, \quad x_{1}^{\prime}=\frac{\xi_{1}^{\prime}}{\vartheta_{1}^{\prime}}, \quad \bar{X}_{1}=\frac{\left(\xi_{1}\right)_{k}}{\left(\vartheta_{1}\right)_{\beta}}=\frac{\left(\xi_{1}^{\prime}\right)_{x}}{\left(\vartheta^{\prime}\right)_{x}},
$$

le superficie $\left(\widehat{X}_{1}\right)$ e $\left(\bar{X}_{1}\right)$ confondendosi ancora nella sfera $\Sigma X^{2}=1$. Lasciando da parte l'effetto ben noto della $B_{k}$ sulla deformata $(50)$ del paraboloide

(1ג) Non comparendo più $a, b$ nel significato attribuito loro al $n^{\circ} 1$, la nuova convenzione non può produrre equiroco. 
$z=x y$, osserviamo che col porre $B(a, b) \equiv B_{k}$ si ha $B(b, a) \equiv B_{k}^{\prime}$, essendo le $B_{k}, B_{k}^{\prime}$ trasformazioni di BranchI a costanti uguali e di classe opposta $\left({ }^{12}\right)$. La principale proprietà geometrica della $B_{k}$ operata sul ciclo di LAPLACE sta in ciò che: Le due congruenze $T$ e $\mathrm{C}$ delle diagonali si trasformano eon trasformazioni asintoliche delle loro falde focali. Per la dimostrazione, già data nella mia Memoria citata in $\left({ }^{6}\right)$, sarà necessario accertarsi che

$$
\left(\xi_{1} \pm h_{1} \xi_{1}^{\prime}, \xi \pm h \xi^{\prime},\left[\xi \pm h \xi^{\prime}\right]_{\alpha},\left[\xi \pm h \xi^{\prime}\right]_{\beta}\right)=0
$$

onde la proprietà, con ciò verificata per le congruenze $\Gamma$ e $\Gamma_{1}$, estendesi alle $C, C_{1}$ in forza della reciprocità rispetto alla sfera.

Rivolgiamoci alla schiera delle superficie $\left(x^{[x]}\right)$ che si collega alla stratificazione della congruenza $\Gamma$ colla $O$ ed associamo l'analoga al ciclo trasformato, scrivendo per ciò conformemente alla formula (53):

$$
x_{1}^{\left[x_{1}\right]}=\frac{\xi_{1}+x_{1} \xi_{1}^{\prime}}{\xi_{1}+x_{1} \xi_{1}^{\prime}}
$$

con $x_{1}$ costante arbitraria. Incominciamo dal mostrare che: Il piano tangente ad una superficie $\left(\mathrm{x}^{[\mathrm{x}]}\right)$ contiene sempre it punto corrispondente di una deter. minata superficie ( $\mathrm{x}_{1}\left[{ }_{\mathrm{x}}\right]$ ), non valendo in generale la reciproca per la stessa coppia di superficie. Basta esprimere la condizione perchè il punto $x_{1}{ }^{\left[x_{1}\right]}$ appartenga al piano $x^{[\mu]} \bar{X} \bar{X}$. Usando coordinate omogenee ed indicando come dianzi la sola prima linea del determinante, abbiamo la equazione:

$$
\left(\xi_{1}+x_{1} \xi_{1}^{\prime}, \xi+x \xi^{\prime}, \xi_{x}, \xi_{\beta}\right)=0
$$

da eui col sussidio delle (55), traiamo la relazione bilineare in $x_{,} x_{1}$ :

$$
x x_{1}+\frac{a}{b}\left(x-x_{1}\right)-1=0, \quad \text { uindi: } \quad x_{1}=\frac{a x-b}{-b x+a} .
$$

Ora, implicando le formule (55) le altre:

$$
\xi=-\frac{2 a b}{t}\left(\xi_{1}\right)_{x}-a \xi_{1}-b \xi_{1}^{\prime}, \quad \xi^{\prime}=-t\left(\xi_{1}^{\prime}\right)_{x}-b \xi_{1}-a \xi_{1}^{\prime},
$$

è palese che la trasformazione inversa è ancora una $B(a, b)$ cogli stessi valori di $a, b$. Riflettendo che la (61) non è simmetrica in $x$ e $x_{1}$, troviamo che la proprietà della congiungente i punti $x^{[x]}$ e $x_{1}^{\left[x_{1}\right]}$ di essere tangente alla superficie $\left(x^{[x]}\right)$ non ha l'analoga rispetto alla $\left(x_{1}\left[x_{1}\right]\right)$. Fanno eccezione $i$ casi in cui si abbia $x=x_{1}=+1$ ovvero $x=x_{1}=-1$, sicehè nel sistema collegato alla

(19) Cangiando $a, b$ ambedue di segno, la trasformazione rimane inalterata. 
stratificazione converrà introdurre come particolarmente notevole la coppia delle superficie:

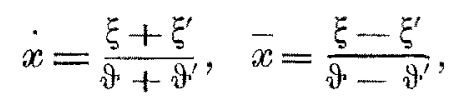

a ciascuna delle quali si associa ordinatamente nel ciclo trasformato l'altra:

$$
\dot{x}_{1}=\frac{\xi_{1}+\xi_{1}^{\prime}}{\vartheta_{1}+{\overrightarrow{\vartheta_{1}^{\prime}}}_{1}^{\prime}}, \quad \vec{x}_{1}=\frac{\xi_{1}-\xi_{1}^{\prime}}{\vec{\vartheta}_{1}-{\overrightarrow{\vartheta_{1}^{\prime}}}_{1}^{\prime}}
$$

Si ha dunque l'importante risultato: Le due rette che si appoggiano alle quattro diagonali del dato ciclo di LAPLACE e del suo trasformato per mezzo della $\mathrm{B}_{\mathrm{k}}$ toccano rispettivamente le coppie di superficie $(\dot{\mathrm{x}}),\left(\dot{\mathrm{x}}_{1}\right)$ e $(\overline{\mathrm{x}}),\left(\overline{\mathrm{x}}_{1}\right)$, luoghi dei loro punti d'incontro colle rette $\mathrm{xx}_{1}$ e $\mathrm{x}_{1} \mathrm{x}^{\prime}{ }_{1}$ di $\Gamma$ e di $\Gamma_{1}$.

Completiamo la proposizione ottenuta provando che: Le due superficie (x) $e \overline{(\mathrm{x})} i$ cui punti dividono armonicamente la diagonale $\mathrm{xx}^{\prime}$ del ciclo di LAPLAOW sono polari reciproche rapporto alla sfera unitaria di centro 0 ; sopra esse le curve $(\alpha, \beta)$ corrispondenti alle reti del ciclo, sono appunto come sulle falde focali le asintotiche, con che il passaggio alle superficie $\left(\dot{x}_{1}\right)$ e $\left(\mathrm{x}_{1}\right)$ connesse al ciclo trasformato si presenta sotto forma di trasformazioni asintotiche simultanee. Dalle (48) infatti si avrà:

$$
\Sigma \dot{x} \bar{x}=1, \quad \Sigma \bar{x} d \dot{x}=0, \quad \Sigma \dot{x} d \bar{x}=0,
$$

onde resta assodata la reciprocita polare. Che le linee $(\alpha, \beta)$ traccino sulla $(x)$ il sistema delle asintotiche, si riconosce osservando che il vettore $\xi-z^{\prime}, \ldots$ ̀े quello della direzione normale alla superficie $(\dot{x})$ e verificando con un sem. plice colcolo che

$$
\Sigma \dot{x}_{\alpha}\left(\xi_{\alpha}-\xi_{\alpha}^{\prime}\right)=0, \quad \Sigma \dot{x}_{\beta}\left(\xi_{\beta}-\xi_{\beta}^{\prime}\right)=0 .
$$

Non è fuor di luogo accennare alle $B_{k}$ singolari con

$$
k= \pm 1 \quad\left(a=1 / \sqrt{2}, \quad b=\mp^{1 / \sqrt{2}}\right),
$$

per cui - sappiamo - si confondono le due classi del Bianchi. Se prendiamo p. es. $a=-b$, troveremo per mezzo delle (55), (61) le formule:

$$
\dot{x}=\frac{\xi+\xi^{\prime}}{\xi+\vartheta^{\prime}}=\frac{h\left(\xi_{1}\right)_{\alpha}+\left(\xi_{1}\right)_{\beta}}{h\left(\vartheta_{1}\right)_{x}+\left(\vartheta_{1}\right)_{\beta}}, \quad \dot{x_{1}}=\frac{\xi_{1}+\xi_{1}^{\prime}}{\xi_{1}+\Re_{1}^{\prime}}=\frac{h_{1} \xi_{\alpha}+\xi_{p}}{h_{1} \vartheta_{\alpha}+\vartheta_{\beta}}, \quad\left(h_{1}=-h t^{2}\right) ;
$$

e formule simili valgono nel caso $a=b$ per $\bar{x}, \bar{x}_{1}$. Di qui : Per le trasformazioni $\mathrm{B}_{\mathrm{k}}$ singolari $\mathrm{k}= \pm 1$ le diagonali non omologhe dei due cicli di LAPLAoE sono complanari; $i$ relativi due punti d'incontro, cioè $i$ punti comuni alle relte $d i \mathrm{\Gamma}$ e di $\mathrm{O}_{1}$ ed a quelle di $\mathrm{O}$ e di $\Gamma_{1}$, percorrono le falde focali di una con. 
gruenza $\mathrm{W}$, la polare reciproca della quale viene descritta dall' intersezione dei rispettivi piani, avente $i$ suoi fuochi sui raggi di $\Gamma$ e di $\Gamma_{1}$. Questo risultato, all' infuori della teoria della trasformazione, sembra notevole, perchè esso stabilisce un nuovo vincolo geometrico tra le congruenza $\Gamma$ e $C$ del ciclo originale. Se ne deducono infatti, note le soluzioni generali dell' equazione di RIccatr (56) per $a= \pm b$, due sistemi di superficie trasformate asintotiche, l'uno della $(\dot{x})$ e l' altro della $(\vec{x})$, giacendo i lóro punti corrispondenti sul raggio $\widehat{X} \bar{X}$ della congruenza $C$; vi si aggiungono le superficie polari i cui piani tangenti passano per la retta $x x^{\prime}$ di $\Gamma$ e che riescono anch'esse trasformate asintotiche delle superficie $(\dot{x})$ e $(\bar{x})$, scambiate fra loro per la reciprocità. Avvertiamo intanto che, col presentarsi d'un siffatto sistema di trasformate asin. totiche, a punti allineati e che pur non si riduca ad un fascio lineare di trasformazioni asintotiche, ci troviamo di fronte ad una configurazione suscettibile di realizzarsi in modo generale per le superficie $R$.

5. Congruenze di diagonali omologhe dei cicli dato e trasformato nella stratificabilita bilaterale. - Riprendiamo lo studio della' $B_{k}$ generale con $a \neq b$ e cerchiamo di esprimere la stratificazione mutua che ammettono, quali congruenze $R$, la $\Gamma$ e la $\Gamma_{1}$, contigue per trasformazioni asintotiche delle falde focali, dispensandoci di stabilire le relazioni analoghe, cioè duali, che varranno per le congruenze $C \cdot e C_{1}$. Assunto sul raggio $x x^{\prime}$ di $\Gamma$ il punto

$$
\Xi=\frac{\xi+\rho \xi^{\prime}}{2+p^{\prime}}
$$

ed esigendo che il piano tangente alla superficie $(\mathbb{E})$ da esso descritta con. tenga la retta corrispondente $x_{1} x_{1}^{\prime}$ di $\Gamma_{1}$, bisognerà che si abbia:

$$
\left(d\left[\xi+\rho \xi^{\prime}\right], \xi_{1}, \xi_{1}^{\prime}, \xi+\rho \xi^{\prime}\right)=0 \text {. }
$$

Ne risulta per la funzione incognita $\rho$ un' equazione a differenziali totali del tipo di RrcoATI, illimitatamente integrabile a causa delle (56):

$$
\rho_{\alpha}=\frac{t}{2 a} \rho^{2}+\left(\frac{a}{t}-\frac{t}{2 b}\right) \rho-\frac{b}{t}, \quad \rho_{\beta}=\frac{a}{h t} \rho^{2}+\left(\frac{h t}{2 a}-\frac{b}{h t}\right) \rho-\frac{h t}{2 b} .
$$

Per quanto riguarda la stratificazione in senso contrario, cioè della congruenza $\Gamma_{1}$ colla $\Gamma$, basterà riportarci alle formule $(61)$ della $B(a, b)$ inversa, onde si ricava: $t_{1}=-2 a b / t$, cosicchè, posto al par della $(64)$ :

$$
\Xi_{1}=\frac{\xi_{1}+p_{1} \xi_{1}^{\prime}}{\vartheta_{1}+p_{1} \vartheta_{1}^{\prime}}
$$


sarà manifesto che nell' attuale processo la $p_{1}$ viene fornita dall' equazione di RICCATr analoga alla (65) :

$$
\left(\rho_{1}\right)_{x}=-\frac{b}{t} \rho_{1}^{2}+\left(\frac{a}{t}-\frac{t}{2 b}\right) \rho+\frac{t}{2 a}, \quad\left(\rho_{1}\right)_{3}=-\frac{a}{h t} \rho_{1}^{2}+\left(-\frac{h t}{2 a}+\frac{b}{h t}\right) \rho_{1}+\frac{h t}{2 b}
$$

Pare notevole che l' una si trasforma nell' altra colla sostituzione lineare:

$$
\rho_{1}=\frac{a \rho-b}{b \rho-a}
$$

Se ne rileva adunque un certo modo di corrispondenza fra le superficie (E) e $\left(\Xi_{1}\right)$ dei due sistemi, pur sussistendo la evidente proprietà che ogni superficie dell' una schiera con una qualunque dell'altra sta in trasformazione asintotica. Questa corrispondenza, col tener presente il significato della (60), potremo interpretare geometricamente colla costruzione seguente: Nota la superficie (E) della prima schiera di stratificazione, cerchisi il punto d' intersezione del piano contenente il punto $\mathrm{\Xi}$ e la retta $\overline{\mathrm{X}} \overline{\mathrm{X}}$ di $\mathrm{C}$ con la diagonale $\mathrm{x}_{1} \mathrm{x}^{\prime}$, del ciclo trasformato, allora il suo coniugato armonico sulla $\mathrm{x}_{1} \mathrm{x}^{\prime}$, descriverà una superficie $\left(\Xi_{1}\right)$ della seconda schiera. Facendo poi:

$$
P_{1}=\frac{t}{T}, \quad \text { quindi : } \quad \rho=\frac{a t-b T}{b t-a T},
$$

ed avendo riguardo alle (56) cui soddisfa la funzione $t$ arriveremo a cangiare le equazioni differenziali (65) nelle seguenti:

$$
T_{z}=-\frac{1}{2 a} T^{2}-\frac{h_{\alpha}}{h} T+b, \quad T_{\beta}=-\frac{h}{2 b} T^{2}+\frac{a}{h},
$$

le quali, come si vede, non sono che le (56) colle costanti $a, b$ invertite e costituiscono per cio la equazione di Rrcoatu da eui dipende la $B(b, a) \equiv B_{k}^{\prime}$. Onde possiamo dire: La stratificazione bilaterale, wente laogo tra le congruenze di diagonali omologhe dei due cicli di LAPLACE che stanno in trasformazioue $\mathrm{B}_{\mathrm{k}}$, viene effettuata adoperando la più generale $\mathrm{B}_{\mathrm{k}}^{\prime}$, cioè colla stessa costante $\mathrm{k}$ e di classe opposta. Tralascieremo di addentrarci nelle conseguenze geometriche, ricordando solo l'importante fatto che dal comporsi la $B_{k}$ colla $R_{k}^{\prime}$ risulta la trasformazione $G_{k}$ data dal GUICHARD.

6. Determinazione delle più generali coppie di snperficie, aventi i loro punti sulle diagonali omologhe dei cicli legati dalla $B_{k}$ e coutigue per trasformazione asintotica. - Dobbiamo anche qui premettere la osservazione che l'argomento eui miriamo, suggerito dai risultati finora conseguiti, e compreso come caso particolare nella teoria delle reti e congruenze $R$. Ritenendo le 
notazioni, introdotte colla $(65)$ in senso più ristretto, designeremo con

$$
\Xi=\frac{\xi+p \xi^{\prime}}{q+p^{\prime}}
$$

ancora un punto del raggio $x x^{\prime}$ di $\Gamma$. Proponiamoci adesso di determinare il fattore $\rho$ in guisa che sulla superficie $(E)$ il doppio sistema delle curve $(\alpha, \beta)$ sia formato dalle asintotiche, corrispondenti così a quelle delle falde focali della $\Gamma$ ed alle reti del ciclo. Notiamo per incidenza, e senza l'intento di farne un uso diretto, che dalle linee $\alpha \pm \beta=$ cost verrà tracciata sulla ( $\Xi$ ) una rete coningata, alla quale, secondo la terminologia di GUICHARD, sarà coniugata la congruenza $\Gamma$, onde desumesi che la intera catena di LAPLACE uscente da questa rete per l'uno e l'altro verso riesce inscritta nella catena aderente alla Г. Ora, siccome deve aversi:

$$
\begin{aligned}
& \left(\left[\xi+\rho \xi^{\prime}\right]_{\alpha x},\left[\xi+\rho \xi^{\prime}\right]_{x},\left[\xi+\rho \xi^{\prime}\right]_{\beta}, \xi+p \xi^{\prime}\right)=0, \\
& \left(\left[\xi+\rho \xi^{\prime}\right]_{\beta \beta},\left[\xi+\rho \xi^{\prime}\right]_{\alpha},\left[\xi+\rho \xi^{\prime}\right]_{\beta}, \xi+\rho \xi^{\prime}\right)=0
\end{aligned}
$$

la funzione richiesta $\rho$ viene assoggettata alle equazioni differenziali:

$$
\begin{aligned}
& \rho_{x \alpha}=\left[\frac{h_{\alpha}}{h}+\frac{2 \frac{\rho}{h}\left(\frac{\rho}{h}\right)_{\alpha}}{\left(\frac{\rho}{h}\right)^{2}-1}\right] \rho_{\alpha}-\frac{2\left(\frac{\rho}{h}\right)_{\alpha}}{\left(\frac{\rho}{h}\right)^{2} 1} \rho_{\beta}+\rho^{2}-1, \\
& \rho_{\beta \beta}=-\frac{2\left(\frac{\rho}{h}\right)_{\beta}}{\left(\frac{\rho}{h}\right)^{2}-1} p_{\beta}+\left[\frac{h_{\beta}}{h}+\frac{2 \frac{\rho}{h}\left(\frac{\rho}{h}\right)_{\beta}}{\left(\frac{\rho}{h}\right)^{2}-1}\right] \rho_{\beta}+\rho^{2}-1,
\end{aligned}
$$

un po complicate, ma che ciò nonostante si prestano al nostro scopo. Ammesso che sieno soddisfatte, avremo in pari tempo:

$$
\begin{aligned}
& \left(\xi+\rho \xi^{\prime}\right)_{\alpha x}=\lambda_{1}\left(\xi+\rho \xi^{\prime}\right)_{x}+\mu_{1}\left(\xi+\rho \xi^{\prime}\right)_{\beta}+\rho\left(\xi+\rho \xi^{\prime}\right) \\
& (\xi+\rho)_{\beta \beta}=\lambda_{2}\left(\xi+\rho \xi^{\prime}\right)_{\alpha}+\mu_{2}\left(\xi+\rho \xi^{\prime}\right)_{\beta}+\rho\left(\xi+\rho \xi^{\prime}\right)
\end{aligned}
$$

$\operatorname{con}$

$$
\lambda_{1}=\frac{h_{\alpha}}{h}+\frac{2 \frac{\rho}{h}\left(\frac{\rho}{h}\right)_{\alpha}}{\left(\frac{\rho}{h}\right)^{2}-1}, \quad \mu_{1}=-\frac{2\left(\frac{\rho}{h}\right)_{\alpha}}{\left(\frac{\rho}{h}\right)^{2}-1}, \quad \lambda_{2}=-\frac{2\left(\frac{\rho}{h}\right)_{\rho}}{\left(\frac{\rho}{h}\right)^{2}-1}, \quad \mu_{2}=\frac{h b}{h}+\frac{2 \frac{\rho}{h}\left(\frac{\rho}{h}\right)_{\beta}}{\left(\frac{\rho}{h}\right)^{2}-1},
$$

Indi, dall' essere $\left(\mu_{1}\right)_{\beta}=\left(\lambda_{2}\right)_{x}$, si conclude: Ogni superficie descritta da un punto di una diagonale del ciclo considerato e che abbia le linee $(\alpha, \beta)$ per asintotiche ̀̀ una superficie $\mathrm{R}$, la relativa rete $\mathrm{R}$ essendo data $d a \quad \alpha \pm \beta=\mathrm{cost}$, ciò̀ corrispondendo alle sviluppabili della congruenza $\mathrm{R}$ formala dalle diagonali. 
Dopo ciò, prescindendo dai casi $\rho= \pm 1$, studiati al numero 4, eseguiamo la sostituzione:

$$
\rho_{x}=\sigma \rho+\tau h, \quad \rho_{\beta}=\tau \rho+\sigma h,
$$

per mezzo della quale le relazioni (72) si convertono nelle più semplici:

$$
\left\{\begin{array}{l}
\sigma_{x} \rho+\tau_{x} h=\sigma^{2} \rho-\frac{h_{\alpha}}{h} \sigma \rho+h \sigma \tau+\rho^{2}-1, \\
\tau_{\beta} \rho+\sigma_{\beta} h=\tau^{2} \rho-\frac{h_{\beta}}{h} \tau \rho+h \sigma \tau+\rho^{2}-1 .
\end{array}\right.
$$

Da queste, sottraendo, si trova:

$$
\left(\sigma_{x}-\tau_{\beta}-\sigma^{2}+\tau^{2}+\frac{h_{x}}{h} \sigma-\frac{h_{\beta}}{h} \tau\right)-h\left(\sigma_{\beta}-\tau_{x}\right)=0,
$$

alla quale si può unire la condizione d'integrabilità delle (73):

$$
h\left(\sigma_{x}-\tau_{\beta}-\sigma^{2}+\tau^{2}+\frac{h_{x}}{h} \sigma-\frac{h_{\beta}}{h} \tau\right)-\rho\left(\sigma_{\beta}-\tau_{x}\right)=0 .
$$

Quindi, poichè $\rho^{2}-h^{2} \neq 0$, supposta la $(\Xi)$ distinta dall' una e l'altra delle due falde focali, otteremo le equazioni:

$$
\sigma_{\beta}=\tau_{\alpha}, \quad \sigma_{\alpha}-\tau_{\beta}-\sigma^{2}+\tau^{2}+\frac{h_{\alpha}}{h} \sigma-\frac{h_{\beta}}{h} \tau=0
$$

La prima di queste ci induce ad assumere una nuova funzione incognita $\varphi^{\prime}$ col porre:

$$
\sigma=-\frac{\varphi_{x}^{\prime}}{\varphi^{\prime}} . \quad \tau=-\frac{\varphi^{\prime} z}{\varphi^{\prime}}
$$

con che la seconda viene a prendere la forma:

$$
\varphi_{x \alpha}^{\prime}+\frac{h_{x}}{h} \varphi_{\alpha}^{\prime}=\varphi_{\beta \beta}^{\prime}+\frac{h_{\beta}}{h} \varphi_{\beta}^{\prime} .
$$

Indicando con $\varphi$ un' altra funzione richiesta, scriviamo:

$$
p=-\frac{\varphi}{\varphi^{\prime}}
$$

onde, derivando col tener conto delle $(73)$ e (78), verrà :

$$
\varphi_{x}=h \varphi_{\beta}^{\prime}, \quad \varphi_{\beta}=h \varphi_{\alpha}^{\prime} .
$$

Ci conviene in fine introdurre una quantità $c$ da riguardarsi previamente 
come funzione di $\alpha, \beta$, facendo in base alle (79):

$$
\varphi_{\alpha x}^{\prime}+\frac{h_{\alpha}}{h} \varphi_{x}^{\prime}=\varphi_{\beta \beta}^{\prime}+\frac{h_{\beta}}{h} \varphi_{\beta}^{\prime}=\varphi+c \varphi^{\prime},
$$

dopo di che l' una o l'altra delle (74) ci fornirà:

$$
\varphi_{\alpha \beta}^{\prime}=\frac{1}{h}\left(\varphi^{\prime}+c \varphi\right) \text {. }
$$

Esprimendo ora le condizioni di integrabilità pel sistema differenziale costituito dalle (80)-(83), troviamo $c_{x}=0, c_{\beta}=0$, quindi: $c=$ cost. Con leggere modficazioni, e cioè attribuendo nel processo analitico così definito la parte principale alla funzione $\varphi$, perverremo a dare al sistema differenziale l'assetto seguente, con $c$ costante arbitraria:

$$
\left\{\begin{array}{l}
\varphi_{\alpha}^{\prime}=\frac{1}{h} \varphi_{\beta}, \quad \varphi_{\beta}^{\prime}=\frac{1}{h} \varphi_{\alpha}, \\
\varphi_{x \alpha}=\frac{h_{\alpha}}{h} \varphi_{x}+c \varphi+\rho^{\prime}, \quad \varphi_{x \beta}=h\left(\rho+c^{\circ} \varphi^{\prime}\right), \quad \varphi_{\beta \beta}=\frac{h_{\beta}}{h} \varphi_{\beta}+c \varphi+\varphi^{\prime},
\end{array}\right.
$$

le (82)-(83) potendo aggiungersi come conseguenze. Qui ancora vale una relazione integrale quadratica:

$$
\frac{2}{h} \varphi_{\alpha} \varphi_{\beta}-\varphi^{2}-\varphi^{\prime 2}-2 c \varphi \varphi^{\prime}=\mathrm{cost} .
$$

Notando che, scelta la $c$, la integrazione del sistema (84) introduce tre costanti disponibili essenziali, cioè i rapporti dei valori iniziali di $\varphi, \varphi^{\prime}, \varphi_{\alpha}, \varphi_{\beta}$, pos. siamo enunciare il risultato colla proposizione: Il problema di determinare sulla diagonale $\mathrm{xx}^{\prime}$ del nostro ciclo di LAPLACE un punto ( $\left.\Xi\right)$ tale che esso descriva una superficie $(\mathbb{\Xi})$ colle asintotiche $(\alpha, \beta)$, corrispondenti alle reti del ciclo, e che per ciò stesso sarà una superficie $\mathrm{R}$, possiede $\infty^{+}$soluzioni, la (E) calcolandosi da $\varphi, \varphi^{\prime}$ nel modo seguente:

$$
\Xi=\frac{\xi \varphi^{\prime}-\xi^{\prime} \varphi}{\vartheta \varphi^{\prime}-\vartheta^{\prime} \varphi}
$$

Ormai riesce facile trarre la conseguenza importante: L' applicazione della $\mathrm{B}_{\mathrm{k}}$, nella quale le funzioni ausiliarie $\varphi, \varphi^{\prime}$ si trasformano appunto come $\xi, \xi^{\prime}, \ldots$ mediante le formule (55), fa passare dalle superficie $(\mathbb{E})$ alla sua omologa $\left(\Xi_{1}\right)$ collegata al ciclo trasformato:

$$
\Psi_{1}=\frac{\xi_{1} \varphi_{1}^{\prime}-\xi_{1}^{\prime} \varphi_{1}}{\vartheta_{1} \varphi_{1}^{\prime}-\vartheta_{1}^{\prime} \varphi_{1}}
$$

la (E) e la $\left(\mathbb{E}_{1}\right)$ essendo contigue per trasformazione asintotica. All' nopo consta- 
tiamo, in primo luogo, ohe le funzioni $\varphi_{1}, \varphi_{1}^{\prime}$, calcolate dalle (55):

$$
\varphi_{1}=t \varphi_{a}-a \varphi-b \varphi^{\prime}, \quad \varphi_{1}^{\prime}=\frac{2 a b}{t} \varphi_{\alpha}^{\prime}-b \varphi-a \varphi^{\prime}
$$

soddisfano al sistema differenziale (84) scritto per l'indice 1, il che ci dimostra che la superficie $\left(\Xi_{1}\right)$ ammette ancora le curve $(\alpha, \beta)$ per asintotiche. Oltre a questo, collo stabilire la relazione:

$$
\left(\left[\xi \varphi^{\prime}-\xi^{\prime} \varphi\right]_{x},\left[\xi \varphi^{\prime}-\xi^{\prime} \varphi\right]_{e}, \xi \varphi^{\prime}-\xi^{\prime} \varphi, \xi_{1} \varphi_{1}^{\prime}-\xi_{1}^{\prime} \varphi_{1}\right)=0
$$

ci si persuade che la retta $\Xi \Xi$, tocea la superficie $(\Xi)$, la medesima proprietia relativa alla superficie $\left(E_{i}\right)$ ricavandosi in modo analogo. Ora, considerando che il punto $\Xi_{1}$ è quello in cui il piano tangente alla $(\mathbb{E})$ viene intersecato dalla retta $x_{1} x_{1}^{\prime}$ di $\Gamma_{1}$, si avrà al tempo stesso la conferma che: Col metodo accennato, cioè integrando il sistema differenziale (84), si ottengono le più generali congruenze $\mathrm{W}$ con $\alpha, \beta$ parametri asintotici delle falde focali ed aventi $i$ rispettivi fuochi sulle rette corrispondenti di $\Gamma$ e di $\Gamma_{1}$.

Per le cose testè esposte dovevansi escludere le particolari superficie (E) trattate al numero 5. A proposito di queste, possiamo aggiungere un'osserva* zione che pare importante: La schiera delle superficie ( $\Xi$ ) con cui viene individuata la stratificazione della congruenza $\Gamma$ con una qualunque $\Gamma_{1}$, apparte. nente quale sua omologa ad un nuovo ciclo di LAPLACE dedotto dall originale per mezzo di una $\mathrm{B}_{\mathrm{k}}$, si determina integrando il sistema differenziale (84) nella condizione speciale che sia nulla la costante che forma il secondo membro della relazione quadratica (85). Affinchè la $\mathrm{B}_{\mathrm{k}}$, applicata al ciclo, risulti reale, occorve che si abbia $|c|>1$. Procedendo alla verifica, facciamo come sopra $\rho=-\varphi / \varphi^{\prime}$. Deriviamo col tener conto delle dne prime (84) ed osserviamo che la supposta relazione omogenea

$$
\frac{2}{h} \varphi_{\alpha} \varphi_{\beta}=\varphi^{2}+\varphi^{\prime 2}+2 c \varphi \varphi^{\prime}
$$

con $2 a b=1 / c, a^{2}+b^{2}=1$, può scriversi nel modo seguente:

$$
\frac{2 \varphi_{\alpha} \varphi \rho}{h \varphi^{2}}=\frac{1}{a b \rho^{2}}(a \rho-b)(b \rho-a)
$$

onde, assunto un fattore $t$, avremo le formule:

$$
\frac{\varphi_{x}}{\rho}=\frac{-\frac{\rho_{x}}{\rho}+\frac{\rho_{\beta}}{h}}{\left(\frac{\rho}{h}\right)^{2}-1}=\frac{1}{t_{\rho}}(a \rho-b), \quad \frac{\varphi_{\rho}}{\varphi}=\frac{\frac{\rho_{\alpha}}{h}-\frac{\rho_{\beta}}{\rho}}{\left(\frac{\rho}{h}\right)^{2}-1}=\frac{t h}{2 a b \rho}(b \rho-\alpha) .
$$

Orbene da queste segue immediatamente la equazione di RIccati (65) per 
la funzione $\rho$, mentre le tre rimanenti relazioni (84) portano all' equazione di Riccatr (56) da soddisfarsi colla $t$ e da cui venne definita l'operazione $B(a, b) \equiv B_{k}$. D'altronde, come era prevedibile, riconosciamo essere la (87) attualmente in difetto, avendosi $\varphi_{1}=0, \varphi^{\prime}=0$.

7. Sistemi di quadrilateri sghembi connessi alle deformate del paraboloide rigato generale. - Le equazioni differenziali (84) alle quali ci ha condotti il problema del numero precedente possono riguardarsi da un altro punto di vista. Dimostreremo che: Dal sistema differenziale (84), nell' ipotesi $|\mathrm{c}|<1$ e per un' opportuna scelta dei valori iniziali, viene a dipendere la costru. zione della superficie applicabili sul paraboloide iperbolico generale, le linee $(\alpha, \beta)$ essendo ancora le asintotiche.

Scriviano, per maggior chiarezza, nuovamente il sistema:

$$
\left\{\begin{array}{l}
\xi_{x}^{\prime}=\frac{1}{h} \xi_{\beta}, \quad \xi_{\beta}^{\prime}=\frac{1}{h} \xi_{\alpha,} \\
\xi_{x, x}=\frac{h_{\alpha}}{h} \xi_{x}+\xi^{\prime}+c \xi, \quad \xi_{\alpha \beta}=h\left(\xi+c \xi^{\prime}\right) . \quad \xi_{\beta \beta}=\frac{h_{\beta}}{h} \xi_{\beta}+\xi^{\prime}+c \xi \\
{\left[\xi_{\alpha x \alpha}^{\prime}=-\frac{h_{\alpha}}{h} \xi_{\alpha}^{\prime}+\xi+c \xi^{\prime}, \quad \xi_{\alpha \beta}^{\prime}=\frac{1}{h}\left(\xi^{\prime}+c \xi\right), \quad \xi_{\beta \beta}^{\prime}=-\frac{h_{\beta}}{h} \xi_{\beta}^{\prime}+\xi+c \xi^{\prime}\right.}
\end{array}\right]
$$

e partiamo da una coppia di solnzioni $\stackrel{\vartheta}{\mathfrak{W}}$ soddisfacente alla relazione:

$$
\frac{2}{h} \vartheta_{2} \vartheta_{\beta}=9^{2}+9^{2}+2 c q^{\prime}+1-c^{2} .
$$

Bisognerà assodare che la forma differenziale quadratica

$$
\Sigma d \widehat{X}^{2}=\frac{1-c^{2}}{\left(\vartheta_{\alpha}^{2}\right)^{2}}\left(d \alpha^{2}+2 \operatorname{chd} d \alpha d \beta+h^{2} d \beta^{2}\right)
$$

generalizzazione della (46), definisce anche qui in modo intrinseco una sfera $(\bar{X})$ di raggio 1. A tal fine, notando che con $|c|<1$ diverrà $e>0, f>0, e g-f^{2}>0$, indicati con $e, f, g$ i coeffficienti della forma (91), esprimiamo la curvatura:

con

$$
K=-\frac{1}{2 \sqrt{e g-f^{2}}}\left(P_{x}+Q_{\beta}\right)
$$

$$
P=\frac{1}{\sqrt{e g-f^{2}}}\left(\frac{1}{2} f \frac{\partial \log \frac{g}{e}}{\partial \beta}+g_{\alpha}-f_{\beta}\right), \quad Q=\frac{1}{\sqrt{e g-f^{2}}}\left(\frac{1}{2} f \frac{\partial \log \frac{e}{g}}{\partial \alpha}+e_{\beta}-f_{\alpha}\right) .
$$

Troveremo:

$$
P=-2 \sqrt{1-c^{2}} \frac{\vartheta^{\prime}}{\hat{V}_{\beta}^{\prime}}, \quad Q=-2 \sqrt{1-c^{2}} \frac{\vartheta^{\prime}}{\vartheta_{\alpha}},
$$


e da qui $K=1$. Conosciuta la sfera $(\hat{X})$ sulla quale, per l'essere $f / \sqrt{e g}=c$, le linee $(\alpha, \beta)$ tracciamo un sistema isogonale, ne deduciamo $\xi, \xi^{\prime}, \eta, \eta^{\prime}, \zeta, \zeta^{\prime}$ mediante le formule seguenti che ora tengono luogo delle (47):

$$
\left\{\begin{array}{l}
\xi+c \xi^{\prime}=\left(\vartheta+c \vartheta^{\prime}\right) \widehat{X}+\vartheta^{\prime} \widehat{X} \\
c \xi+\xi^{\prime}=\left(c \vartheta^{\prime}+\vartheta^{\prime}\right) \bar{X}+q_{\alpha} \widehat{X}_{x},
\end{array}\right.
$$

in guisa da completare il gruppo delle soluzioni linearmente indipendenti del sistema (84). Dalle (92), la somma estendendosi alle tre lettere, discendono le:

$$
\Sigma \xi^{2}=\vartheta^{2}+1, \quad \Sigma \xi^{\prime 2}=\mathfrak{Y}^{\prime 2}+1, \quad \Sigma \xi \xi^{\prime}=\vartheta \vartheta^{\prime}-c
$$

mentre la seconda e la terza riga delle (48) restano inalterate:

$$
\begin{aligned}
& \left(\Sigma \xi^{\prime} d \xi=\mathscr{\vartheta}^{\prime} d \vartheta, \quad \Sigma\left(\xi_{x}\right)^{2}=\left(\vartheta_{\alpha}\right)^{2}, \quad \Sigma\left(\xi_{\beta}\right)^{2}=\left(\vartheta_{\beta}\right)^{2}\right. \\
& \text { i }\left[\Sigma \xi d \xi^{\prime}=q d \mathcal{G}^{\prime}, \quad \Sigma\left(\xi_{\alpha}^{\prime}\right)^{2}=\left(q_{\alpha}^{\prime}\right)^{2}, \quad \Sigma\left(\xi_{\beta}^{\prime}\right)^{2}=\left(q_{\beta}^{\prime}\right)^{2}\right] \text {. }
\end{aligned}
$$

Osserviamo che qui ancora risulta:

$$
\bar{X}=\frac{\xi_{\alpha}}{\vartheta_{\alpha}}=\frac{\xi_{\beta}^{\prime}}{\mathfrak{V}_{\beta}^{\prime}}, \quad \bar{X}=\frac{\xi_{\beta}}{\vartheta_{\beta}}=\frac{\zeta_{\alpha}^{\prime}}{\vartheta_{\alpha}^{\prime}},
$$

il punto $\widetilde{X}$ giacendo sulla stessa sfera unitaria $\Sigma X^{2}=1$, ed assegniamo come superiormente sull' intersezione dei piani tangenti i due punti:

$$
x=\frac{\xi}{2}, \quad x^{\prime}=\frac{\xi^{\prime}}{y^{\prime}} .
$$

Conserveremo le notazioni $\Gamma$ e $C$ per le congruenze delle rette $x x^{\prime}$ e $\widehat{X} \bar{X}$.

Prima di discutere la figura formata di $T$ e $C$, giova constatare che contemporaneamente viene fornita da tre quadrature

$$
x^{*}=\int\left(\xi^{\prime} d q+\xi d q^{\prime}\right)
$$

una superficie $\left(x^{*}\right)$ d'elemento lineare

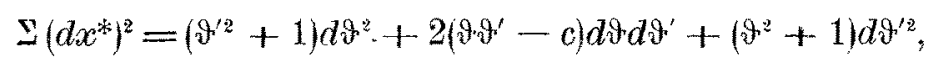

la quale ̀̀ dunque l'annunciata deformata del paraboloide rigato generale:

$$
\left\{\begin{array}{l}
x_{0}=\sqrt{\frac{1-c}{2}}\left(q+y^{\prime}\right), \quad y_{0}=\sqrt{\frac{1+c}{2}}\left(q-y^{\prime}\right), \quad z_{0}=y g^{\prime}, \\
\frac{x_{0}^{2}}{1-c}-\frac{y_{0}^{2}}{1+c}=2 z_{0} .
\end{array}\right.
$$

Sembra superfluo riportar qui la verifica, priva di difficolta, che riguarda le asintotiche. Avvertiamo però che, restando la deformazione di prima specie, il sistema coniugato permanente sarà pure dato da $\alpha \pm \beta=$ cost; esso corri- 
sponde alle sviluppabili delle congruenze $\Gamma$ e $C$. Sussiste peraltro la proprietà che i raggi $\bar{X}$ e $\bar{X}$ della sfera di centro $O$ sono paralleli all' asse del paraboloide nel rotolamento sull' una e l'altra faccia della deformata.

Ci resta da esaminare il modo secondo cai si scompone nell' attuale generalizzazione il nostro ciclo di LapcaCe, legato esclusivamente al caso del paraboloide iperbolico equilatero. Vediamo anzitutto che si conserva la stratificabilità unilaterale della congruenza $\Gamma$ con la $C$, come anche la formula $(53)$ che definisce la schiera delle superficie $\left(x^{[}[x]\right)$. Ora, fissando due qualunque valori di $x$, ̀̀ bene evidente che il quadrilatero sghembo $x^{\left[x_{1}\right]} \widehat{X}_{x}\left[x_{0}\right] \bar{X}$ verrà sem. pre a descrivere un sistema dotato della proprietà che $i$ lati sono tangenti comuni alle superficie luogo dei vertici. Fra questi sistemi ne esistono due, in generale distinti e confondentisi per $c=0$ nel ciclo di LAPLAOE, quelli cioè generati dai quadrilateri $x \bar{X} x^{\prime} \bar{X}$ e $x^{[6]} \bar{X} x^{[1 / \epsilon]} \bar{X}$, avendosi :

$$
x^{[c]}=\frac{\xi+c \xi^{\prime}}{\vartheta+c \vartheta^{\prime}}, \quad x^{[1 / c]}=\frac{c \xi+\xi^{\prime}}{c \vartheta^{\prime}+\mathscr{\vartheta}^{\prime}} .
$$

Si consideri difatti sopra le superficie $(x)$ e $\left(x^{\prime}\right)$ i doppii sistemi di curve $(\alpha, \beta)$, oramai non coniugati. Le loro tangenti non omologhe, come facilmente desumesi dalle (95), (96), concorrono nei punti $\bar{X}$ e $\bar{X}$ della sfera. In connessione del tutto analoga stanno $i$ sistemi isogonali $(\alpha, \beta)$ segnati dai punti $\widehat{X}$ e $\widehat{X}$ sulla sfera, essendo $x^{[1 / c]}$ e $x^{[c]}$ i punti d'incontro delle tangenti, il che si dimostra subito per mezzo delle (92). E da notarsi intanto che ambedue i sistemi appartengono ad una classe importante ed abbastanza generale, presentandosi quando per una coppia di superficie coincidano i piani osculatori non omologhi alle curve $(\alpha, \beta)$ di riferimento. Tale classe, secondo un teorema stabilito nella mia Memoria citata nella nota ( $)$, gode della notevole proprietà che le rette congiungenti i punti e formanti in tempo stesso le intersezioni dei piani osculatori percorrono una congruenza $W$ con $\alpha, \beta$ parametri asin. totici delle falde focali; cio che vale adunque per le congruenze $\Gamma$ e $C$ in discorso. In quanto alla giacitura relativa dei due quadrilateri, aggiungiamo la osservazione che le quattro tangenti alla sfera uscenti rispettivamente da ciascuno dei punti $\widehat{X}$ e $\bar{X}$ si ordinano in due coppie mutualmente perpendicolari. Per convincersene, senza calcoli, basta riflettere che p. es. la $\bar{X} x$ e la $\widehat{X} x^{[1 / \epsilon]}$, di direzione $x_{\%}$ e $\bar{X}_{\%}$, sono tangenti alle curve sferiche corrispondenti alle sviluppabili della congruenza di raggio $\bar{X} x$, per ciò coniugate, vale a dire ortogonali. Avvertiamo da ultimo che qui ancora le congruenze $\Gamma$ e $C$ sono $R$ e che le sei quantità

$$
\vartheta \xi^{\prime}-\vartheta^{\prime} \xi, \ldots, \eta \xi^{\prime}-\xi_{\eta}^{\prime}, \ldots
$$


(circolando. le lettere $\xi$, $\eta$, $)$ figurano come coordinate omogenee di retta per la congruenza $\Gamma$, le due terne seambiandosi fra di loro, con opportuna scelta di segno, nel passaggio alla $C$. La equazione di LAPLACE soddisfatta dalle coordinate di rette, conformemente al menzionato teorema di DarBoux, risulta del tipo di Moutard:

$$
\Theta_{\mu \beta}=\left(h+\frac{1}{h}\right) \Theta
$$

coincidendo così con quella associata alle asintotiche della deformata $\left(x^{*}\right)$, per le quale la direzione della normale e data dal vettore $\eta \xi^{\prime}-\xi \eta^{\prime}$. Riguardo all' altra terna $\sqrt{ } \xi^{\prime}-\$^{\prime} \xi, \ldots$ si constata che essa rappresenta la direzione nor. male alla superficie ausiliaria:

$$
\tilde{x}=x^{*}-\vartheta \xi^{\prime}-\Re^{\prime} \xi
$$

riferita del pari alle esintotiche $(\alpha, \beta)$ e che viene generata dal punto d'intersezione del piano taugente alla deformata $\left(x^{*}\right)$ coll'asse del paraboloide rotolante. Il legame geometrico esistente rispettivamente fra le superficie $\left(x^{*}\right),(\tilde{x})$ e le congruenze $C$ e $\Gamma$ è quella ben nota reciprocitò di superficie $\mathrm{R}$ e congruenza $\mathrm{R}$, caratterizzata dal parallelismo della normale all' una colla retta dell'altra, proprietà che sussiste, e cioè invertendosi, per le tangenti alla rete $R$, formanti congruenze $R$, e le falde focali della congruenza $R$, formanti superficie $R$, in modo da mantenersi indefinitamente nell' applicazione successiva della trasfor* mazione di LAPLACE.

8. Determinazione delle coppie di sistemi sferici corrispondentisi con coincidenza dei piani osculatori non omologhi. La nltima parte della nostra ricerca sarà dedicata ad un problema che è spontaneamente suggerito dai risultati or ora conseguiti: Trovare $i$ piu generali doppii sistemi di curve, tracciati sopra una medesima sfera e per cui si confondano i piani osculatori non omologhi ed in conseguenza $i$ rispettivi circoli osculatori. Riusciremo a stabilire che il caso segnalato al numero 7 ne abbraccia la soluzione completa, la proposizione da dimostrare enunciandosi così: I sistemi sferici richiesti sono tutti e soli quelli dedotti dalle deformate del paraboloide rigato, costruibili col rappresentare le asintotiche $(\alpha, \beta)$ sulla sfera per mezzo di raggi paralleli all' asse del paraboloide rotolante sull' una e l'altra faccia.

Per la verifica, alquanto lunga, riesce utile studiare in primo lnogo la rappresentazione della sfera sopra se stessa per la quale sull' intersezione dei piani tangenti vi abbia almeno un punto descrivente una superficie il cui piano tangente passi per la corda di contatto. Indichiamo coi vettori $X$ e $X^{\prime}$, funzioni di una medesima coppia di variabili, i due punti della sfera $\Sigma X^{*}=1$, 
effettuando cosi un cambiamento di notazione in vista della maggiore generalità dell'attuale questione. il punto generico $x$ sull' intersezione dei due piani tangenti può esprimersi mediante un parametro $\sigma$ nel modo seguente:

$$
x=\frac{1}{2 \cos ^{2} \theta}\left[X+X^{\prime}-\sigma\left(Y Z^{\prime}-Z Y^{\prime}\right)\right], \quad \cos 2 \theta=\mathbf{v} X X^{\prime} .
$$

Affinchè il piano $x X X^{\prime}$, colla direzione normale data dal vettore:

$$
\sigma\left(X+X^{\prime}\right)+Y Z^{\prime}-Z Y^{\prime}
$$

diventi piano tangente ad una superficie $(x)$, occorre che si abbia:

$$
\frac{d \sigma}{\sigma^{2}+1}=\frac{1}{\sin ^{2} 2 \theta} \Sigma\left(Y Z^{\prime}-Z Y^{\prime}\right)\left(d X+d X^{\prime}\right)
$$

Di qui, ammessa la integrabilità ed essendo $\sigma^{0}$ una soluzione particolare:

$$
\sigma=\frac{\sigma^{0}+O}{1-C \sigma^{0}} \quad(C=\cos t)
$$

Dunque: Perchè nella rappresentazione della sfera sopra se stessa esista una superficie $(x)$ colle rette $x X$ e $x X^{\prime}$ tangenti comuni alla sfera ed alla $(x)$, e necessario e sufficiente che l' espressine

$$
\frac{1}{1-\left(\Sigma X X^{\prime}\right)^{2}}\left|\begin{array}{lll}
d X+d X^{\prime}, & X, & X^{\prime} \\
d Y+d Y^{\prime}, & Y, & Y^{\prime} \\
d Z+d Z^{\prime}, & Z, & Z^{\prime}
\end{array}\right|
$$

risulti un differensiale esatto. Ma allora vi è nna semplice infinitc̀ di tali superficie, sicchè la congruenza generata dall' intersezione dei piani tangenti sarà stratificabile unilateralmente con quella delle corde di contatto $X X^{\prime}\left({ }^{13}\right)$. Due qualunque delle rispettive tangenti alla sfera uscenti dal punto $X$ o dal punto $X^{\prime}$ fanno angolo costante, ciò che si verifica in base alla (102), essendo:

$$
\begin{gathered}
\cos \Omega=\frac{\Sigma(x-X)\left(x^{0}-X\right)}{\sqrt{\Sigma(x-X)^{2} \cdot \sqrt{\Sigma\left(x^{0}-X\right)^{2}}}}=\frac{\sigma \sigma^{0}+1}{\sqrt{\sigma^{2}+1} \sqrt{\left(\sigma^{0}\right)^{2}+1}}= \\
=\frac{ \pm 1}{\sqrt{\sigma^{2}+1}}, \quad \pm 1=\operatorname{sgn}\left(1-C \sigma^{0}\right) .
\end{gathered}
$$

(13) La condizione equivale a quella avvertita nella Memoria citata nelia nota $\left({ }^{2}\right)$ pel caso della quadrica riferita ai parametri delle generatrici rettilinee, corrispondendo $u, v$ e $u^{\prime}, v^{\prime}$ ai dne puntí, la quale consiste in ciò che

deve essere differenziale esatto.

$$
\frac{d u}{u^{\prime}-u}-\frac{d v}{v^{\prime}-v}
$$


Ora, considerando che il piano $x X X^{\prime}$ è osculatore alla curva sferica toccata dalla retta $X x$ e che an primo sistema di tali curve sferiche potrà scegliersi ad arbitrio, riconosceremo che: La rappresentazione richiesta dalla sfera sopra se stessa può operarsi mediante le rette intersezioni dei piani osculatori di un qualsiasi doppio sistema isogonale di curve sferiche.

Dopo ciò, per risolvere il problema propostoci al principio del presente numero, designeremo con $\widehat{X}, \bar{X}$ i punti della sfera descriventi due sistemi $(\alpha, \beta)$, esigendo dunque che per questi si confondano i piani osculatori non omologhi. Segue da quanto fu previamente osservato che tali sistemi sono isogonali e che vale la stratificabilita della congruenza $\Gamma$ colla $C$. Inoltre, col riportarei all'argomentazione atilizzata al numero 7, concludiamo che anche le superficie $(x)$ e $\left(X^{\prime}\right)$, luoghi dei punti d'incontro delle tangenti alla sfera, uscenti dai punti $\bar{X}$ e $\bar{X}$ ortogonalmente a quella dei sistemi $(\alpha, \beta)$, appartengono alla schiera della stratificazione e che, di più, le curve di $(x)$ e di $\left(x^{\prime}\right)$, toccate da queste tangenti, costituiscono ancora due sistemi $(x, \beta)$ a piani osculatori comnni. Con ciò il nostro problema si riduce all'altro: Costruire le coppie di superficie $(\mathrm{x})$ e $\left(\mathrm{x}^{\prime}\right)$, riferite alle curve $(\alpha, \beta)$, per cui concorrono le tangenti non omologhe verso $i$ due punti $\widehat{\mathrm{X}}$ e $\overline{\mathrm{X}}$ della sfera unitaria di centro 0 , pur essa toccata da queste langenti.

Facciamo come manifestamente è lecito:

$$
\begin{gathered}
x=\frac{\xi}{2}, \quad x^{\prime}=\frac{\xi^{\prime}}{y^{\prime}} . \\
\mathbf{\Sigma} \xi^{2}=\xi^{\prime 2}+1, \quad \Sigma \xi^{\prime 2}=g^{\prime 2}+1,
\end{gathered}
$$

notando che, in virtù delle (105), i valori assolutori di $1 / \sigma$ e di $1 / \sigma^{*}$ significano i segmenti tangenziali rispettivamente fra i punti $x$ e $x^{\prime}$ e la sfera $\Sigma X^{2}=1$. Affinchè la retta $x \widehat{X}$ sia tangente alla sfera ed in tempo stesso tangente di direzione $x_{\alpha}$ alla $(x)$, deve aversi:

$$
\Sigma \widehat{X} \xi=\uparrow, \quad \widehat{X}=\frac{\xi}{\vartheta}+\rho\left(\frac{\xi}{\vartheta}\right)_{x},
$$

indi per effetto della (105): $\rho=\$ / \mathcal{W}_{\alpha}$. Proseguendo questo ragionamento, giungeremo a stabilire le formule:

$$
\widehat{X}=\frac{\xi_{x}}{\vartheta_{\alpha}}=\frac{\xi_{\beta}^{\prime}}{\mathfrak{\vartheta}_{\beta}^{\prime}}, \quad \bar{X}=\frac{\xi_{\beta}}{\vartheta_{\beta}}=\frac{\xi_{\alpha}^{\prime}}{\hat{\vartheta}_{x}^{\prime}},
$$

completate dalle relazioni:

$$
\Sigma\left(\xi_{x}\right)^{2}=\left(\theta_{x}\right)^{2}, \quad \Sigma\left(\xi_{\beta}\right)^{2}=\left(q_{\beta}\right)^{2} .
$$

In forza della $(106)$ si arrà in $\xi, \eta, \zeta$, ำ:

$$
\xi_{\alpha}=h \xi_{\beta}^{\prime}, \quad \xi_{p}=\bar{h} \xi_{\alpha}^{\prime} .
$$


Derivate le (105) e tenuto conto delle (108), verrà:

$$
\Sigma^{\prime} d \xi=\Psi^{\prime} d y^{\prime}, \quad \Sigma \xi d \xi^{\prime}=2 d \mathscr{W}^{\prime}
$$

di qui integrando:

$$
\Sigma \xi \xi^{\prime}=y y^{\prime}-c
$$

con $c$ costante. Avvertasi intanto che $|c|<1$, poichè, indicando $\omega$ l'angolo formato dalle rette $\widehat{X} x \quad \widehat{X} x^{\prime}$, risulta con semplice calcolo: $\cos \omega= \pm c$, $\pm 1=-\operatorname{sgn}\left(\mathfrak{H} \vartheta^{\prime}\right)$; evidentemente è da escludersi l'ipotesi $|c|=1$.

Dobbiamo ora esprimere le derivate seconde di $\xi, \eta, \zeta$, $\$$ per mezzo delle quaderne $\xi, \xi^{\prime}, \xi_{x}, \xi_{\beta}$ ecc., il determinante $\delta=\left(\xi, \xi^{\prime}, \xi_{x}, \xi_{\beta}\right)$ non potendo esssere nullo. Per tale scopo torna agevole l'uso del sommatorio $S$ esteso alle quattro lettere, l'ultimo termine prendendosi col segno mutato, onde invece delle (105) (110), (109), (107) scriveremo più brevemente.

(111) $S \xi^{2}=1, \quad S \xi^{\prime 2}=1, \quad S \xi \xi^{\prime}=-c, \quad S \xi^{\prime} d \xi=0, \quad S\left(\xi_{x}\right)^{2}=0, \quad S\left(\xi_{\beta}\right)^{2}=0$.

Aggiungiamo le relazioni dedotte per derivazione:

$$
S \xi_{\alpha x} \xi=S \xi_{\alpha x} \xi_{\alpha}=S \xi_{\alpha \beta} \xi^{\prime}=S \xi_{\beta \beta} \xi=S \xi_{\beta \beta} \xi_{\beta}=0 .
$$

Notando che con $\delta \neq 0$ si ha necessariamente $S \xi_{\alpha} \xi_{\beta} \neq 0$, le formule volute, deducibili dalle (112), presentano l'aspetto seguente:

$$
\xi_{\alpha x}=\lambda^{\prime} \xi_{x}+\lambda\left(\xi^{\prime}+c \xi\right), \quad \xi_{\alpha \beta}=\mu\left(\xi+c \xi^{\prime}\right), \quad \xi_{\beta \beta}=v^{\prime} \xi_{\beta}+\nu\left(\xi^{\prime}+c \xi\right)
$$

e concordano così col fatto che i piani $x \widehat{X} x^{\prime}$ e $x \bar{X} x^{\prime}$ riescono piani osculatori comuni alle curve $(\alpha, \beta)$ descritte dai punti $x$ e $x^{\prime}$. Occorre paragonare alle (113) la relazione tratta dall' uguagliare i due valori di $\xi_{x \beta}$ calcolati dalle (108), il che ci darà:

$$
\lambda^{\prime}=\frac{h_{\alpha}}{h}, \quad \nu^{\prime}=\frac{\tilde{h}_{\beta}}{\bar{h}}, \frac{\lambda}{\nu}=\frac{h}{\bar{h}} .
$$

Dopo di eiò, per condurre a termine l'attuale ricerca, basterebbe formare le condizioni di integrabilità pel sistema (113); dovremmo però, come ci si per* suade col ealcolo effettivo, supporre $c \neq 0$. Per evitare tale restrizione, giova prima invocare le relazioni:

$$
S \xi_{\alpha} \xi_{\beta}=-S \xi_{\alpha \beta} \xi=-\mu\left(1-c^{2}\right), \quad S \xi_{\alpha} \xi^{\prime}=0, \quad S \xi_{\beta} \xi^{\prime}=0
$$

da cui, derivando la seconda e la terza rispettivamente rapporto a $\alpha$ ed a $\beta$, avremo:

$$
S \xi_{\alpha, \gamma} \xi^{\prime}-\frac{1}{h} S \xi_{x} \xi_{\beta}=0, \quad S \xi_{\beta \beta} \xi^{\prime}-\frac{1}{h} S \xi_{\alpha} \xi_{\beta}=0
$$


indi :

$$
\lambda=\frac{\mu}{h}, \quad \nu=\frac{\mu}{h} .
$$

Delle equazioni fornite dalle condizioni di integrabilita conviene notare in primo luogo le due: $\lambda_{\beta}=0, \nu_{x}=0$. Esse, coll' aver riguardo alle (113) rendono manifesto che si può dịsporre delle variabili $\alpha, \beta$ in modo da ridurre $\lambda$ e $v$ all' unità positiva o negativa. Anzi di più, cangiando all' occorrenza i segni di $\xi^{\prime}$ e di $c$, sard ammissibile di fare p. es. $\nu=1$ e porre $\lambda=\varepsilon^{\prime}= \pm 1\left(^{1+}\right)$. Segue allora dalla terza (114): $\bar{h}=\varepsilon^{\prime} h$. Resta finalmente come sola condizione da soddisfare la equazione a derivate parziali imposta alla funzione $h$ :

$$
(\log h)_{\chi \beta}=h-\frac{\varepsilon^{\prime}}{h} \text {. }
$$

Vale adunque il sistema differenziale sotto la sua forma definitiva:

$$
\left\{\begin{array}{l}
\xi_{\alpha}^{\prime}=\frac{\varepsilon^{\prime}}{h} \xi_{\beta}, \quad \xi_{\beta}^{\prime}=\frac{1}{h} \xi_{\alpha}, \\
\xi_{\alpha \alpha \alpha}=\frac{h_{\alpha}}{h} \xi_{\alpha}+\varepsilon^{\prime}\left(\xi^{\prime}+c \xi\right), \quad \xi_{\alpha \beta}=h\left(\xi+c \xi^{\prime}\right), \quad \xi_{\beta \beta}=\frac{h_{\beta}}{h} \xi_{\beta}+\xi^{\prime}+c \xi, \\
{\left[\xi_{\alpha \alpha \alpha}^{\prime}=-\frac{h_{\alpha}}{h} \xi_{\alpha}^{\prime}+\varepsilon^{\prime}\left(\xi+c \xi^{\prime}\right), \quad \xi_{\alpha \beta}^{\prime}=\frac{\varepsilon^{\prime}}{h}\left(\xi^{\prime}+c \xi\right), \quad \xi_{\beta \beta}^{\prime}=-\frac{h_{\beta}}{h} \xi_{\beta}^{\prime}+\xi_{\xi}+c \xi^{\prime}\right]}
\end{array}\right.
$$

coll'aggiunta delle relazioni (111), compatibili, poichè, ammesso $\varepsilon^{\prime}=+1$, abbiamo ritrovato appunto il sistema (91), (93), (94) del numero 7. Osserviamo d' altronde che la estensione al caso $\varepsilon^{\prime}=+1$ risponde soltanto all' altra ipoteri, ivi trascurata, che le sviluppabili delle congruenze $\Gamma$ e $C$ risultino immaginarie coniugate, date allora da $\alpha \pm i \beta=$ cost, vale a dire che l' associata deformazione del paraboloide rigato (99) sia di seconda specie. Così trovasi compiuta la prova della nostra asserzione.

(4) Distinguendo per tale denotazione la $\varepsilon^{t}= \pm 1$ dalla $\varepsilon= \pm 1$ che figura con allro significato nel sistema $(26)$ del $n^{0} \mathbf{2}$. 